\title{
The HAMMONIA Chemistry Climate Model: Sensitivity of the Mesopause Region to the 11-Year Solar Cycle and $\mathrm{CO}_{2}$ Doubling
}

\author{
H. Schmidt, G. P. Brasseur, M. Charron,* E. Manzini, ${ }^{+}$M. A. Giorgetta, and T. Diehl \# \\ Max Planck Institute for Meteorology, Hamburg, Germany \\ V. I. FOMICHEV \\ York University, Toronto, Ontario, Canada \\ D. Kinnison, D. Marsh, And S. Walters \\ National Center for Atmospheric Research, Boulder, Colorado
}

(Manuscript received 23 January 2005, in final form 14 December 2005)

\begin{abstract}
This paper introduces the three-dimensional Hamburg Model of the Neutral and Ionized Atmosphere (HAMMONIA), which treats atmospheric dynamics, radiation, and chemistry interactively for the height range from the earth's surface to the thermosphere (approximately $250 \mathrm{~km}$ ). It is based on the latest version of the ECHAM atmospheric general circulation model of the Max Planck Institute for Meteorology in Hamburg, Germany, which is extended to include important radiative and dynamical processes of the upper atmosphere and is coupled to a chemistry module containing 48 compounds. The model is applied to study the effects of natural and anthropogenic climate forcing on the atmosphere, represented, on the one hand, by the 11-yr solar cycle and, on the other hand, by a doubling of the present-day concentration of carbon dioxide. The numerical experiments are analyzed with the focus on the effects on temperature and chemical composition in the mesopause region. Results include a temperature response to the solar cycle by 2 to 10 $\mathrm{K}$ in the mesopause region with the largest values occurring slightly above the summer mesopause. Ozone in the secondary maximum increases by up to $20 \%$ for solar maximum conditions. Changes in winds are in general small. In the case of a doubling of carbon dioxide the simulation indicates a cooling of the atmosphere everywhere above the tropopause but by the smallest values around the mesopause. It is shown that the temperature response up to the mesopause is strongly influenced by changes in dynamics. During Northern Hemisphere summer, dynamical processes alone would lead to an almost global warming of up to $3 \mathrm{~K}$ in the uppermost mesosphere.
\end{abstract}

\section{Introduction}

The mesosphere-lower thermosphere (MLT) region, which covers the approximate altitude range 50-150 $\mathrm{km}$, is one of the most complex, yet least explored lay-

\footnotetext{
* Current affiliation: Meteorological Service of Canada, Dorval, Canada.

+ Current affiliation: National Institute for Geophysics and Volcanology, Bologna, Italy.

\# Current affiliation: NASA Goddard Space Flight Center, Greenbelt, Maryland.
}

Corresponding author address: H. Schmidt, Max Planck Institute for Meteorology, Bundesstrasse 53, 20146 Hamburg, Germany.

E-mail: hauke.schmidt@zmaw.de ers of the atmosphere. Rocket soundings and satellite monitoring have highlighted the strong variations that occur as a function of height in the physics and in the chemical composition, especially in the vicinity of the mesopause between 85- and 100-km altitudes (Smith 2004). For example, as the altitude increases through this atmospheric region, the strong vertical mixing conditions that maintain the mixing ratio of long-lived species relatively constant with height are progressively replaced by conditions in which molecular diffusion prevails, leading to gravitational separation of chemical species according to their respective mass.

The rapid change with height in the amount of solar radiation available to photolyze atmospheric molecules also leads to a rapid chemical transition between the mesosphere and the thermosphere, with molecules 
dominating the atmospheric composition at the lower levels and atomic species becoming most abundant in the upper layers.

The nature of the energy budget is also changing with altitude. Below $90 \mathrm{~km}$, the major contributions are provided by solar heating resulting from ozone absorption, by the emission of infrared radiation by $\mathrm{CO}_{2}$, by adiabatic processes, and by the transport of heat through advection. In the lower thermosphere, the energy budget is determined primarily by the absorption of shortwave solar radiation by molecular oxygen, by radiative emissions by $\mathrm{CO}_{2}$, and by downward molecular conduction of heat. In the MLT region, the pattern is considerably more complicated. As a consequence of the low collision frequency between particles, the thermal radiative transfer starts to be affected by nonthermodynamic equilibrium (NONLTE) conditions. Additionally, processes such as energetic particle input, chemical heating, airglow emissions, and turbulent heat exchanges must be taken into account (Mlynczak 2000; Roble 2000; Fomichev et al. 2002).

The deposition of momentum from upwardpropagating waves provides the major forcing mechanism for the meridional circulation of the middle atmosphere. The dissipation of gravity waves produces a body force on the zonal flow, leading even to a reversal of the zonal wind in the vicinity of the mesopause. Simultaneously, this momentum source produces a mesospheric circulation directed from the summer pole to the winter pole, and through mass continuity gives rise to ascending and descending motions in the summer and winter hemispheres, respectively (e.g., Holton and Alexander 2000). Other wave motions have strong effects on the structure of the middle atmosphere, including diurnal and semidiurnal tides, which produce periodic fluctuations in temperature, wind components and species concentrations, planetary waves, and equatorial waves that are (together with gravity waves) believed to drive the observed semiannual and quasi-biennial oscillations (e.g., Garcia 2000; Giorgetta et al. 2002). In all cases, the source of the waves is located in the lower atmosphere or at the earth's surface, and their ability to reach the mesopause region depends on filtering and dissipation mechanisms that take place at lower altitudes. In the thermosphere, where the degree of ionization becomes significant, electromagnetic forces, which tend to align the motions of charged particles along magnetic field lines, affect atmospheric motions, so that the resistance effect of ions on the neutral matter (usually named "ion drag") must be considered in the dynamical equations.

The dynamical structure of the mesopause region therefore depends simultaneously on solar energy that is progressively absorbed by the atmospheric medium as it penetrates downward, and momentum sources provided by waves originating from the lower layers of the atmosphere and absorbed as they propagate upward and dissipate or encounter critical levels. Natural variations and human-induced changes to be expected in the MLT region cannot be assessed without understanding the dynamical, radiative, and chemical couplings existing between the different layers of the atmosphere. Therefore, as pointed out by Roble (2000), there is a need to increase the height of the boundary of current middle-atmosphere models well into the thermosphere and include solar and auroral processes as well as other physical and chemical processes such as ion drag, molecular diffusion, molecular thermal conductivity, and electrodynamic interactions with the ionosphere.

The Hamburg Model for the Neutral and Ionized Atmosphere (HAMMONIA), which is presented here, has been designed to investigate how the couplings between atmospheric regions affect the response of the atmosphere to external perturbations, including solar variability and anthropogenic chemical emissions at the earth's surface. A particularly challenging endeavor is to determine the depth of penetration of the signal produced by solar variability in the atmosphere. Another interesting challenge is to quantify the impact on the upper atmosphere of the release at the surface of large quantities of carbon dioxide and other radiatively active gases. Addressing these questions requires that the chemical processes of importance in the atmosphere be treated fully interactively with the dynamical, physical, and radiative processes included in the model.

Most contemporary general circulation models (GCMs) extend typically to approximately $2-10 \mathrm{hPa}$, with the stratospheric layers being considered as a buffer between the tropopause and the top of the model. Some of these GCMs have been extended to approximately 75-100-km altitude (e.g., Fels et al. 1980; Boville 1995; Hamilton et al. 1995; Manzini et al. 1997; Beagley et al. 1997) or even up to the thermosphere (Miyahara et al. 1993; Fomichev et al. 2002; Sassi et al. 2002). Chemical transport models that treat chemical processes up to the mesosphere "offline" from the dynamics have also been developed (e.g., Chipperfield et al. 1993; Brasseur et al. 1997). Coupled dynamicalchemical models covering this altitude range used mostly a mechanistic approach (e.g., Rose and Brasseur 1989; Lefèvre et al. 1994; Sonnemann et al. 1998) in which the complex processes of the troposphere are replaced by boundary conditions (e.g., planetary wave forcing) applied in the vicinity of the tropopause. In 


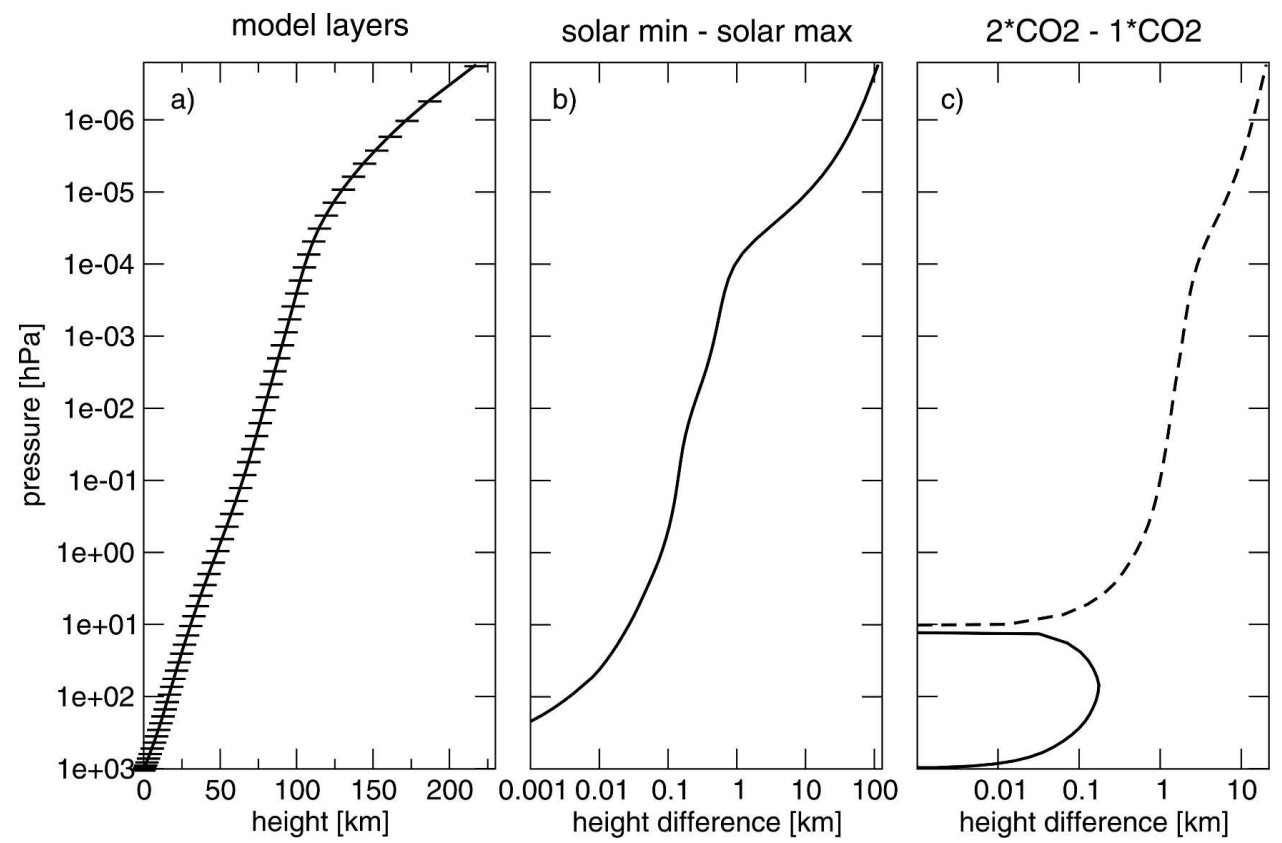

FIG. 1. The annual mean global average relation of pressure and geometrical height in the model. (a) Simulated in the solar minimum run. Horizontal dashes indicate the centers of the 67 model layers. (b) Simulated height change at given pressure levels for the solar maximum run with respect to solar minimum. (c) Simulated height change for the $2 \times \mathrm{CO}_{2}$ run with respect to $1 \times \mathrm{CO}_{2}$. The dashed line marks negative values.

more recent times GCMs have been coupled "online" to chemistry, some of them having the lid in the mesosphere (e.g., Rasch et al. 1995; Steil et al. 2003) or close to the mesopause (de Grandpré et al. 2000). Only very recently, some GCMs with coupled chemistry extending from the surface to the thermosphere have been developed or are under development. This is the case for the Extended Canadian Middle Atmosphere Model (CMAM; e.g., Fomichev et al. 2002), for the Whole Atmosphere Community Climate Model (WACCM; e.g., Sassi et al. 2002) developed at the National Center for Atmospheric Research in Boulder, Colorado, and for HAMMONIA, initiated at the Max Planck Institute (MPI) for Meteorology in Hamburg, Germany, with the contribution of scientists in other research institutions.

The purpose of this paper is to present and describe the newly developed HAMMONIA (section 2), and to evaluate calculated atmospheric fields (temperature, winds, and chemical species) by comparison with available observational data (section 4). The model is then used to calculate the response of the atmosphere to the 11-yr solar cycle (section 5) and to a doubling in the atmospheric concentration of $\mathrm{CO}_{2}$ (section 6). A discussion of the results and some conclusions are presented in section 7 .

\section{Model description}

HAMMONIA consists of the vertical extension to the thermosphere of the MAECHAM5 model (Giorgetta et al. 2006; Manzini et al. 2006), which is itself a vertical extension to the mesosphere of the ECHAM5 atmospheric general circulation model (Roeckner et al. 2003, 2005, 2006). ECHAM5 is the most recent version in a series of ECHAM models evolving originally from the spectral weather prediction model of the European Centre for Medium-Range Weather Forecasts (ECMWF; Simmons et al. 1989) and includes, in particular, comprehensive descriptions of the energy budget, the water cycle, and land surface processes. The ECHAM model versions have been applied to a large variety of climate research issues.

HAMMONIA is a spectral model with triangular truncation at wavenumber 31 (T31) and with 67 vertical levels ranging from the surface to $1.7 \times 10^{-7} \mathrm{hPa}(\sim 250$ $\mathrm{km})$. Figure 1 shows the distribution of the vertical levels. In the mesosphere and lower thermosphere the distance between the levels is constant in log-pressure and corresponds to about 2 to $3 \mathrm{~km}$, depending on temperature. The model includes a full dynamic and radiative coupling with the Model of Ozone and Related Tracers (MOZART3) chemical module (Kinnison et al. 2006, 
manuscript submitted to J. Geophys. Res.). For integration of the dynamical and chemical processes we adopt a time step of $10 \mathrm{~min}$.

The dynamical and radiative processes that have been specifically implemented in HAMMONIA include solar heating in the ultraviolet and extreme ultraviolet wavelength regime (down to $5 \mathrm{~nm}$ ), a NONLTE radiative scheme, energy deposition and eddy diffusion generated by gravity wave breaking, vertical molecular diffusion and conduction, and a simple parameterization of electromagnetic forces in the thermosphere (ion drag and Lorenz forces).

\section{a. Radiative heating and cooling}

The parameterizations adopted to quantify radiative heating and cooling are up to certain pressure levels and for large parts of the solar spectrum identical to the ones used in ECHAM5. In particular, the Rapid Radiative Transfer Model (RRTM) for longwave radiation (Mlawer et al. 1997) and an updated version of the shortwave parameterization of Fouquart and Bonnel (1980) that divides the spectrum from 250 to $4000 \mathrm{~nm}$ into four bands are employed. Additional formulations are introduced to account for processes specific to the MLT region: solar heating at shorter wavelengths, NONLTE effects in radiative processes, and chemical heating. A similar approach of merging different heating and cooling schemes as detailed in the following was already employed by Fomichev et al. (2002).

Infrared cooling by $\mathrm{O}_{3}$ and $\mathrm{CO}_{2}$ above $0.02 \mathrm{hPa}(\sim 75$ $\mathrm{km})$ is calculated from the parameterization of Fomichev and Blanchet (1995) and of Fomichev et al. (1998), respectively, with NONLTE effects explicitly taken into account for the $\mathrm{CO}_{2}$ cooling. The latter parameterization was modified so that the interpolation of matrix coefficients is based on $\mathrm{CO}_{2}$ column amounts rather than on local $\mathrm{CO}_{2}$ densities. This modification allows the use of nonconstant $\mathrm{CO}_{2}$ profiles also below $95 \mathrm{~km}\left(\sim 50 . \times 10^{-4} \mathrm{hPa}\right)$. Below $0.1 \mathrm{hPa}(\sim 65 \mathrm{~km})$ infrared radiative fluxes are computed purely by RRTM. Between 0.1 and $0.02 \mathrm{hPa}$, the infrared radiative fluxes are calculated as a linear combination of both schemes. A parameterization of solar heating by $\mathrm{CO}_{2}$ absorption in the near infrared according to the NONLTE scheme of Ogibalov and Fomichev (2003) is utilized. This contribution is particularly important at heights of around $75 \mathrm{~km}(\sim 0.02 \mathrm{hPa})$, where it can account for up to $30 \%$ of the total radiative heating (Fomichev et al. 2004b). The values from the parameterization are ignored below $30 \mathrm{hPa}[\sim 24 \mathrm{~km}$, because heating in the near infrared is already considered by the scheme of Fouquart and Bonnel (1980)], fully considered above $3 \mathrm{hPa}(\sim 40 \mathrm{~km})$, and merged with the near- infrared $\mathrm{CO}_{2}$ heating provided by the scheme of Fouquart and Bonnel (1980) between these two pressure levels.

Two slightly different combinations of solar heating parameterizations are used in the experiments of this study, hereafter called SW1 and SW2. In SW1, solar heating at wavelengths from 680 to $2500 \mathrm{~nm}$ is computed in three bands using the parameterization of Fouquart and Bonnel (1980) as in ECHAM5. The fourth band of the Fouquart and Bonnel (1980) parameterization $(250$ to $680 \mathrm{~nm})$ is used only up to $70 \mathrm{hPa}(\sim 18$ $\mathrm{km})$. Above $30 \mathrm{hPa}(\sim 24 \mathrm{~km})$, heating by $\mathrm{O}_{3}$ and $\mathrm{O}_{2}$ at wavelengths from 120 to $680 \mathrm{~nm}$ is computed using the same algorithm as for the calculation of the photodissociation rates (see section 2e), and noting that, if $J_{\lambda, x}$ is the photolysis rate for a molecule $x$ and a wavelength $\lambda$, the corresponding rate of energy deposition is given by $E_{\lambda, x}=h \nu J_{\lambda, x}$, with $\nu$ being the frequency of the radiation and $h$ being Planck's constant. Between 30 and 70 $\mathrm{hPa}$, the two approaches are merged. In SW2, the fourth band of the Fouquart and Bonnel (1980) parameterization is used for all pressure levels. Only between 120 and $250 \mathrm{~nm}$ are heating and photodissociation rate computation done consistently. The rationale of these approaches is that SW1 with its significantly better resolution in the UV part of the spectrum is much better suited to represent the spectral dependence of solar variability and is therefore used in the solar cycle experiments of this study. SW2, on the other hand, is very similar to the approach used in the original ECHAM5 model. It allows an easier comparison to earlier ECHAM5 experiments and is used in the $\mathrm{CO}_{2}$ doubling experiments of this study. At thermospheric heights, an extreme ultraviolet (EUV) solar forcing based on the model of Richards et al. (1994) is used for wavelengths ranging from 5 to $105 \mathrm{~nm}$. Efficiency factors to account for the loss of internal energy due to airglow processes are taken from Mlynczak and Solomon (1993) for the $\mathrm{O}_{3}$ Hartley band and $\mathrm{O}_{2}$ Schumann-Runge continuum, and from Roble (1995) for the EUV portion of the spectrum. The solar EUV heating efficiency also accounts for energy losses associated with radiative cooling in the $5.3-\mu \mathrm{m} \mathrm{NO}$ band. Although this is not an uncommon approach, it should be taken into account in the interpretation of the experiments in this study as a potential source of errors in particular during polar night in the upper atmosphere. With the exception of the parameterization of Fouquart and Bonnel (1980), all heating schemes consider heating also at solar zenith angles larger than $90^{\circ}$. However, the Fouquart and Bonnel (1980) scheme is important only in the troposphere and in parts of the stratosphere, and according 
to Fomichev et al. (2004a) the sphericity adds no noticeable contribution to the total heating below the stratopause.

Chemical heating is computed within the chemistry scheme for seven exothermic reactions as given by Brasseur and Offermann (1986). The loss of energy by chemiluminescence is taken into account using efficiency factors from Mlynczak and Solomon (1993). Chemical heating is the release of heat by recombination reactions between atoms or radicals produced as a result of photolysis. This contribution is particularly important at heights where the photodissociation products can travel large distances before recombining. To avoid double counting the incoming radiative energy, in the Tropospheric Ultraviolet and Visible Radiation Model (TUV) code, the energy corresponding to the photodissociation energy threshold $E_{B, x}$ is subtracted from the energy deposition rate: $E_{\lambda, x}=\left(h \nu-E_{B, x}\right) J_{\lambda, x}$ with $x$ being $\mathrm{O}_{3}$ or $\mathrm{O}_{2}$. For the heating computed by the scheme of Fouquart and Bonnel (1980) in SW2, we assume that, in this part of the spectrum, $23 \%$ of the total incoming energy is converted to chemical potential energy.

The parameterizations of radiative processes require that the concentrations of the following compounds be known: $\mathrm{O}_{3}, \mathrm{O}_{2}, \mathrm{O}\left({ }^{3} \mathrm{P}\right)$ (used in the infrared cooling scheme in the deactivation process of $\left.\mathrm{CO}_{2}\right), \mathrm{CO}_{2}, \mathrm{H}_{2} \mathrm{O}$, $\mathrm{CH}_{4}, \mathrm{~N}_{2}, \mathrm{~N}_{2} \mathrm{O}, \mathrm{CFCs}$, and aerosols. The two latter groups are taken from climatologies representing present-day conditions. For all other compounds the prognostic model concentrations are used. The specific heat $c_{p}$ and the gas constant $R$ are computed at each time step according to the calculated concentrations of chemical compounds. This is of importance in height regions above about $90 \mathrm{~km}\left(\sim 2 \times 10^{-3} \mathrm{hPa}\right)$ where the major atmospheric constituents are not uniformly mixed.

\section{b. Parameterized gravity wave tendencies}

Orographic gravity wave drag and surface blocking are parameterized using Lott and Miller (1997), as in ECHAM5. The momentum flux deposition from a spectrum of nonorographic gravity waves is parameterized as in MAECHAM5. In addition, in HAMMONIA the parameterizations of the heating and the eddy diffusion of constituents, momentum, and temperature generated by the breaking of gravity waves are taken into account following the parameterization of Hines (1997a,b). The viscous correction to the eddy diffusion coefficient suggested by Akmaev et al. (1997) is applied. It reduces eddy coefficients computed by the Hines scheme at altitudes where molecular mixing is strong. HAMMONIA also differs from MAECHAM5 in the specification of the gravity wave source spectrum. In HAMMONIA, this spectrum includes waves from tropospheric frontal activity (Charron and Manzini 2002) and a lower boundary for the vertical wavenumber spectrum. The parameterized gravity wave tendency from nonorographic sources is strongly sensitive to the choice of a cutoff vertical wavenumber. After test simulations with different choices of this parameter, it has been decided not to allow vertical wavenumbers smaller than $2 \pi /(12 \mathrm{~km})$ in order to obtain realistic mesospheric winds and temperatures.

\section{c. Molecular diffusion and conduction}

The radiative balance in the lower thermosphere region is dominated by the extreme ultraviolet solar heating and the downward transport of heat by molecular processes. Hence, molecular viscosity and conductivity are of primary importance in this region of the upper atmosphere. The transport and vertical distribution of constituents are also strongly affected by molecular viscosity and diffusion.

Vertical molecular diffusion of constituents (labeled $i$ ) in the vertical direction follows the governing equation (Huang et al. 1998):

$$
\frac{\partial X_{i}}{\partial t}=\frac{1}{\rho} \frac{\partial}{\partial z}\left(\rho D_{i} \frac{\partial X_{i}}{\partial z}\right)-\frac{1}{\rho} \frac{\partial}{\partial z}\left(\rho w_{D i} X_{i}\right) .
$$

Here, $X_{i}$ is the mass mixing ratio of constituent $i, D_{i}$ is the respective diffusion coefficient $\left(m^{2} s^{-1}\right)$, and $w_{D i}$ is the drift vertical velocity of constituent $i$, which acts to separate constituents of different molar masses. Using the hydrostatic equation and performing a variable change from altitude coordinates to pressure coordinates, the diffusion equation can be rewritten as

$$
\frac{\partial X_{i}}{\partial t}=g \frac{\partial}{\partial p}\left(g \rho^{2} D_{i} \frac{\partial X_{i}}{\partial p}\right)+g \frac{\partial}{\partial p}\left(\rho w_{D i} X_{i}\right) .
$$

A semi-implicit vertical discretization that conserves mass is used to solve Eq. (2) for each constituent $i$. The semi-implicit factor is chosen to be 1.5. The diffusion coefficient (multiplied by density, $\rho D_{i}$ ) and the vertical drift velocity (multiplied by density, $\rho w_{D i}$ ) are taken from Banks and Kockarts (1973):

$$
\begin{aligned}
\rho D_{i} & =4.17 \times 10^{-6}\left(\frac{T}{273.15 K}\right)^{0.5}\left(m_{A}+\frac{m_{A}^{2}}{m_{i}}\right)^{0.5} \\
\rho w_{D i} & =\frac{\rho D_{i} g}{R^{*} T}\left[m_{A}-m_{i}+\alpha_{T i} \rho R^{*}\left(\frac{\partial T}{\partial p}\right)\right],
\end{aligned}
$$

where $m_{A}$ is the molar mass of air $\left(\mathrm{g} \mathrm{mol}^{-1}\right), m_{i}$ is the molar mass of constituent $i\left(\mathrm{~g} \mathrm{~mol}^{-1}\right), R^{*}=8.31436 \mathrm{~J}$ $\left(\mathrm{mol}^{-1} \mathrm{~K}^{-1}\right)^{-1}$, and $\alpha_{\mathrm{T} i}$ is the expansion coefficient for constituent $i$ ( -0.38 for $H$, zero for all other constitu- 
ents). It has to be noted that after diffusion of the constituents, the sum of their mass mixing ratios is normalized to unity, because there is no other constraint to locally conserve this sum in the numerical treatment of molecular diffusion. Another option would be to solve the diffusion equations for number densities rather than mixing ratios (see Chabrillat et al. 2002). Both methods have been tested offline, and results were almost identical.

The vertical molecular diffusion of momentum is performed as for constituents, except that

$$
\begin{aligned}
X_{i} & \rightarrow \mathbf{u}=(u, v) \\
\left(\rho D_{i}\right) & \rightarrow \mu=1.87 \times 10^{-5}\left(\frac{T}{273.15 \mathrm{~K}}\right)^{0.69} \\
\left(\rho w_{\mathrm{D} i}\right) & \rightarrow 0,
\end{aligned}
$$

where $\mu$ is chosen to be the viscosity of atomic oxygen (Banks and Kockarts 1973), since this atom is the dominant component in the uppermost model layers where the importance of molecular diffusion becomes largest.

Diffusion of heat uses similar equations in which

$$
\begin{aligned}
& \mathbf{u} \rightarrow T \\
& \mu \rightarrow \mu / \operatorname{Pr} .
\end{aligned}
$$

Here, $\operatorname{Pr}=0.72$ is the Prandtl number observed for air (Batchelor 1967).

Frictional heating is also taken into account (the molecular diffusion of momentum leads to energy deposition in the form of heat). The temperature tendency due to frictional heating is written as (Gill 1982)

$$
\left(\frac{\partial T}{\partial t}\right)_{\text {frict. }}=\frac{g^{2} \rho \mu}{c_{p}}\left[\left(\frac{\partial u}{\partial p}\right)^{2}+\left(\frac{\partial v}{\partial p}\right)^{2}\right] .
$$

\section{d. Ion drag and Lorenz force}

In the MLT region, the neutral mass flow is affected by the presence of ionized gases that follow the magnetic field of the earth. In HAMMONIA, this effect is parameterized following the simple Hong and Lindzen (1976) approach, in which the impact on the neutral flow can be represented by a drag and a force normal to the flow (the Lorenz force). Therefore, the wind tendency caused by ion drag $(D)$ and Lorenz force $(L)$ is expressed as

$$
\begin{aligned}
& \frac{\partial u}{\partial t}=-D u-(L \sin \beta) v \\
& \frac{\partial v}{\partial t}=-\left(D \sin ^{2} \beta\right) v+(L \sin \beta) u
\end{aligned}
$$

where $\tan \beta=-2 \tan \phi$, if $\phi$ is the latitude. The values of $D$ and $L$ are given by Hong and Lindzen (1976) as a function of geopotential height. The leapfrog time discretization is performed using a semi-implicit scheme for stability reasons, with the semi-implicit factor chosen to be 1.5 .

The ion drag is also producing heating. The kinetic energy dissipated by ion drag is introduced into the diabatic term of the temperature equation.

It should be noted that this parameterization gives only a relatively crude mean estimation of the thermospheric ion drag. It is planned that future model versions include a detailed treatment of E-region ions to allow for a more realistic response of the ion drag to the variability of solar irradiance and geomagnetic activity.

\section{e. Chemistry}

HAMMONIA uses a condensed version of the MOZART3 chemistry module (Kinnison et al. 2006, manuscript submitted to J. Geophys. Res.) with 48 compounds, 46 photolyses, (Table 1) $107 \mathrm{bi}$, and termolecular gas phase reactions (Table 2). While the adopted tropospheric chemical scheme is limited to the $\mathrm{CH}_{4}-$ $\mathrm{NO}_{x}-\mathrm{HO}_{x}-\mathrm{O}_{3}$ system and ignores therefore the effects of nonmethane hydrocarbons, this reaction scheme represents in suitable detail the neutral chemistry of importance in the middle and upper atmosphere. Heterogeneous reactions associated with the formation of the ozone hole in the lower polar stratosphere are not included in the present study. The chemical system is solved with a 10-min time step, using the fully implicit Euler backward scheme with Newton-Raphson iteration. An explicit algorithm is utilized for the long-lived species $\left(\mathrm{CH}_{4}, \mathrm{~N}_{2} \mathrm{O}, \mathrm{CO}, \mathrm{CO}_{2}, \mathrm{CH}_{3} \mathrm{Cl}, \mathrm{CH}_{3} \mathrm{Br}, \mathrm{CFCl}_{3}\right.$, $\mathrm{CF}_{2} \mathrm{Cl}_{2}, \mathrm{CFCl} 13, \mathrm{HCFC} 22, \mathrm{CCl}_{4}, \mathrm{CH}_{3} \mathrm{CCl}_{3}, \mathrm{CF}_{3} \mathrm{Br}$, and $\mathrm{CF}_{2} \mathrm{ClBr}$ ).

The computation of photodissociation rates is based on the TUV scheme (Madronich and Flocke 1998) with the clear-sky rates tabulated for 122 bands in the wavelength interval extending from 200 to $780 \mathrm{~nm}$. The tabulation is performed with respect to geopotential height, surface albedo, $\mathrm{O}_{3}$ column amount, and the solar zenith angle. The part of the spectrum ranging from 120 to 200 $\mathrm{nm}$ is treated explicitly with 34 wavelength intervals and uses parameterizations for the photolysis of $\mathrm{O}_{2}$ from Chabrillat and Kockarts (1998; Lyman- $\alpha$ ), Brasseur and Solomon (1986; Schumann-Runge continuum), and Koppers and Murtagh (1996; SchumannRunge bands). The NO photolysis is treated according to Minschwaner and Siskind (1993).

In the thermosphere, $\mathrm{NO}$ is produced mainly as a consequence of the dissociation of molecular nitrogen involving ambient or energetic electrons. The latter can be either of auroral origin or produced through photoionization processes. A more detailed description of the 
TABLE 1. Photolyses process included in the model.

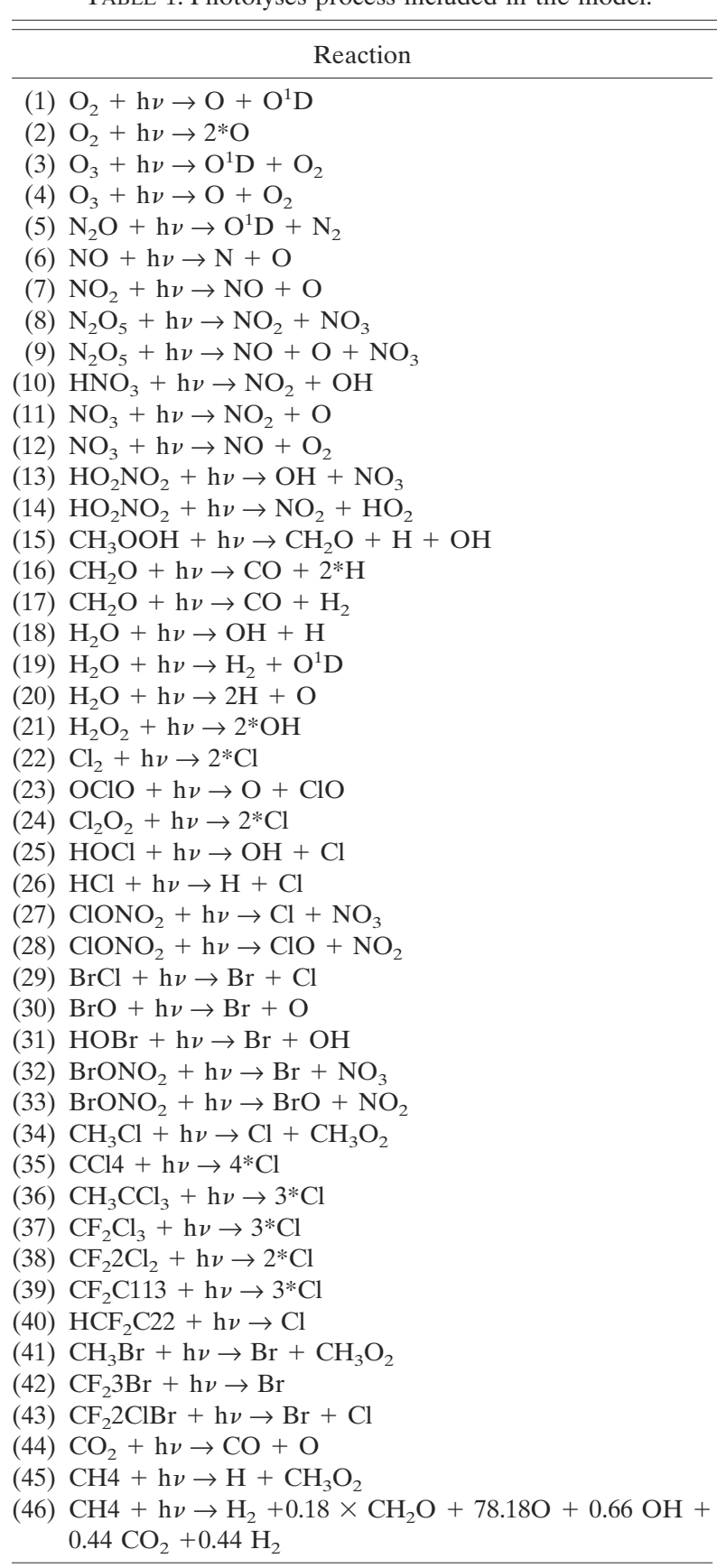

thermospheric NO production is given, for example, by Bailey et al. (2002). In this first version of HAMMONIA, which does not treat the ionosphere explicitly, the thermospheric NO production is parameterized following the work of Huang et al. (1998) and McEwan and Phillips (1975). The parameters used by Huang et al. (1998) have been adapted to obtain thermospheric NO concentrations that are close to recent observations with the Student Nitric Oxide Explorer (SNOE) satellite in- strument (Barth et al. 2003). The auroral production is derived by assuming that the earth's magnetic North Pole is located at geographic coordinates $\left(78.5^{\circ} \mathrm{N}\right.$, $291.0^{\circ} \mathrm{E}$ ). We compute an additional NO production in the stratosphere as a consequence of ion production by cosmic rays following Heaps (1978), and assuming that one NO molecule is produced per generated ion pair. An option for future model versions would be to apply the more detailed parameterization of thermospheric NO production provided by Marsh et al. (2004).

For most of the constituents, concentrations at the lower boundary are specified at the surface as monthly mean values as calculated by the MOZART2 model (Horowitz et al. 2003) for present-day conditions. Only $\mathrm{CO}_{2}$ and $\mathrm{N}_{2} \mathrm{O}$ are not taken from MOZART2 but fixed to volume mixing ratios of 360 (720) ppmv and 315 pptv, respectively. The upper boundary is assumed to be a rigid lid for most compounds with the exception of atomic hydrogen $(\mathrm{H})$ and oxygen $\left(\mathrm{O}^{3} \mathrm{P}\right)$. For these two constituents, the concentrations in the top layer are relaxed to values provided by the extended Mass Spectrometer Incoherent Scatter (MSISE-90) climatology (Hedin 1991).

Chemical species are updated each time step by a sequence of operators: advection, molecular diffusion, turbulent diffusion, cloud processes, and chemistry. Cloud processes include convection and the phase transitions of water. Advection of tracers is performed using the flux form semi-Lagrangian scheme of Lin and Rood (1996).

\section{Setup of the simulations}

To assess the effect of the 11-yr solar cycle two simulations with a length of $20 \mathrm{yr}$ each have been performed using the SW1 solar heating approach (see section 2a). Both simulations are performed for "present-day" conditions of greenhouse gas concentrations as described above. The first one represents conditions typical of a solar cycle minimum (as observed in September 1986). This simulation is also used in the validation of the model (section 4). The second simulation represents conditions typical of solar maximum (November 1989). The following parameters differ in the two runs.

- Solar irradiance from 120 to $300 \mathrm{~nm}$ as shown in Fig. 2. Differences between 300 and $700 \mathrm{~nm}$ are also considered but in general are well below $1 \%$. The values were provided by J. Lean (2004, personal communication). Data for the part of the spectrum from 200 to $400 \mathrm{~nm}$ are described by Lean et al. (1997).

- The changes in the EUV radiation are represented according to the parameterization of Richards et al. 
TABLE 2. Gas phase chemical reactions included in the model and corresponding reaction rates. Read 6.e-34 as 6.*10 ${ }^{-34}$; am: air density (number per $\mathrm{cm}^{3}$ ); rate coefficients are expressed in $\mathrm{cm}^{3} \mathrm{~s}^{-1}$ for bimolecular and $\mathrm{cm}^{6} \mathrm{~s}^{-1}$ for termolecular reactions. Troe: Rate coefficients for Troe reactions are given as $k=\left[k_{o} / 1+\left(k_{o} \times a m\right) / k_{i}\right] \times 0.6\left\langle 1+\left\{\log \left[\left(k_{o} \times a m / k_{i}\right)\right]^{2}\right\}^{-1}\right\rangle$.

\begin{tabular}{|c|c|}
\hline Reaction & $\begin{array}{l}\text { Rate coefficient } \\
\text { (from MOZART3 chemical module) }\end{array}$ \\
\hline (1) $\mathrm{O}+\mathrm{O}_{2}+\mathrm{M} \rightarrow \mathrm{O}_{3}+\mathrm{M}$ & $6 . \mathrm{e}-34 *(300 / \mathrm{T}) 2.4$ \\
\hline (2) $\mathrm{O}+\mathrm{O}_{3} \rightarrow 2 * \mathrm{O}_{2}$ & $8.00 \mathrm{e}-12 * \exp (-2060 / \mathrm{T})$ \\
\hline (3) $\mathrm{O}+\mathrm{O}+\mathrm{M} \rightarrow \mathrm{O}_{2}+\mathrm{M}$ & $4.23 \mathrm{e}-28 / \mathrm{T} 2$ \\
\hline (4) $\mathrm{O}+\mathrm{H}_{2} \rightarrow \mathrm{OH}+\mathrm{H}$ & $7.00 \mathrm{e}-11 * \exp (-5130 / \mathrm{T})$ \\
\hline (5) $\mathrm{O}^{1} \mathrm{D}+\mathrm{N}_{2} \rightarrow \mathrm{O}+\mathrm{N}_{2}$ & $1.80 \mathrm{e}-11 * \exp (110 / \mathrm{T})$ \\
\hline (6) $\mathrm{O}^{1} \mathrm{D}+\mathrm{O}_{2} \rightarrow \mathrm{O}+\mathrm{O}_{2}$ & $3.20 \mathrm{e}-11 * \exp (70 / \mathrm{T})$ \\
\hline (7) $\mathrm{O}^{1} \mathrm{D}+\mathrm{H}_{2} \mathrm{O} \rightarrow 2 * \mathrm{OH}$ & $2.20 \mathrm{e}-10$ \\
\hline (8) $\mathrm{O}^{1} \mathrm{D}+\mathrm{N}_{2} \mathrm{O} \rightarrow 2 * \mathrm{NO}$ & $6.70 \mathrm{e}-11$ \\
\hline (9) $\mathrm{O}^{1} \mathrm{D}+\mathrm{N}_{2} \mathrm{O} \rightarrow \mathrm{N}_{2}+\mathrm{O}_{2}$ & $4.9 \mathrm{e}-11$ \\
\hline (10) $\mathrm{O}^{1} \mathrm{D}+\mathrm{O}_{3} \rightarrow \mathrm{O}_{2}+\mathrm{O}_{2}$ & $1.20 \mathrm{e}-10$ \\
\hline (11) $\mathrm{O}^{1} \mathrm{D}+\mathrm{CF}_{2} 2 \mathrm{Cl}_{2} \rightarrow 2 * \mathrm{Cl}$ & $1.20 \mathrm{e}-10$ \\
\hline (12) $\mathrm{O}^{1} \mathrm{D}+\mathrm{CF}_{2} \mathrm{C} 113 \rightarrow 3^{*} \mathrm{Cl}$ & $1.50 \mathrm{e}-10$ \\
\hline (13) $\mathrm{O}^{1} \mathrm{D}+\mathrm{HCF}_{2} \mathrm{C} 22 \rightarrow \mathrm{Cl}$ & $7.20 \mathrm{e}-11$ \\
\hline (14) $\mathrm{O}^{1} \mathrm{D}+\mathrm{CH}_{4} \rightarrow \mathrm{CH}_{3} \mathrm{O}_{2}+\mathrm{OH}$ & $1.13 \mathrm{e}-10$ \\
\hline (15) $\mathrm{O}^{1} \mathrm{D}+\mathrm{CH}_{4} \rightarrow \mathrm{CH}_{2} \mathrm{O}+\mathrm{H}+\mathrm{HO}_{2}$ & $3.00 \mathrm{e}-11$ \\
\hline (16) $\mathrm{O}^{1} \mathrm{D}+\mathrm{CH}_{4} \rightarrow \mathrm{CH}_{2} \mathrm{O}+\mathrm{H}_{2}$ & $7.50 \mathrm{e}-12$ \\
\hline (17) $\mathrm{O}^{1} \mathrm{D}+\mathrm{H}_{2} \rightarrow \mathrm{H}+\mathrm{OH}$ & $1.10 \mathrm{e}-10$ \\
\hline (18) $\mathrm{O}^{1} \mathrm{D}+\mathrm{HCl} \rightarrow \mathrm{Cl}+\mathrm{OH}$ & $1.50 \mathrm{e}-10$ \\
\hline (19) $\mathrm{N}_{2} \mathrm{D}+\mathrm{O}_{2} \rightarrow \mathrm{NO}+\mathrm{O}$ & $5.00 \mathrm{e}-12$ \\
\hline (20) $\mathrm{N}_{2} \mathrm{D}+\mathrm{O} \rightarrow \mathrm{N}_{2}+\mathrm{O}$ & $4.50 \mathrm{e}-13$ \\
\hline (21) $\mathrm{N}+\mathrm{O}_{2} \rightarrow \mathrm{NO}+\mathrm{O}$ & $1.50 \mathrm{e}-11 * \exp (-3600 / \mathrm{T})$ \\
\hline (22) $\mathrm{N}+\mathrm{NO} \rightarrow \mathrm{N}_{2}+\mathrm{O}$ & $2.10 \mathrm{e}-11 * \exp (100 / \mathrm{T})$ \\
\hline (23) $\mathrm{NO}+\mathrm{O}+\mathrm{M} \rightarrow \mathrm{NO}_{2}+\mathrm{M}$ & $\begin{array}{l}\text { troe: } \mathrm{k}_{O}=9.00 \mathrm{e}-32 *(300 / \mathrm{T})^{1.5} \\
\mathrm{~K}_{i}=3.00 \mathrm{e}-11\end{array}$ \\
\hline (24) $\mathrm{NO}+\mathrm{HO}_{2} \rightarrow \mathrm{NO}_{2}+\mathrm{OH}$ & $3.50 \mathrm{e}-12 * \exp (250 / \mathrm{T})$ \\
\hline (25) $\mathrm{NO}+\mathrm{O}_{3} \rightarrow \mathrm{NO}_{2}+\mathrm{O}_{2}$ & $3.00 \mathrm{e}-12 * \exp (-1500 / \mathrm{T})$ \\
\hline (26) $\mathrm{NO}_{2}+\mathrm{O} \rightarrow \mathrm{NO}+\mathrm{O}_{2}$ & $5.60 \mathrm{e}-12 * \exp (180 / \mathrm{T})$ \\
\hline (27) $\mathrm{NO}_{2}+\mathrm{O}+\mathrm{M} \rightarrow \mathrm{NO}_{3}+\mathrm{M}$ & $\begin{array}{l}\text { troe: } \mathrm{k}_{O}=9.00 \mathrm{e}-32 *(300 / \mathrm{T})^{2} \\
\mathrm{~K}_{i}=2.20 \mathrm{e}-11\end{array}$ \\
\hline (28) $\mathrm{NO}_{2}+\mathrm{O}_{3} \rightarrow \mathrm{NO}_{3}+\mathrm{O}_{2}$ & $1.20 \mathrm{e}-13 * \exp (-2450 / \mathrm{T})$ \\
\hline (29) $\mathrm{NO}_{2}+\mathrm{NO}_{3}+\mathrm{M} \rightarrow \mathrm{N}_{2} \mathrm{O}_{5}+\mathrm{M}$ & $\begin{array}{l}\text { troe: } \mathrm{k}_{O}=2.00 \mathrm{e}-30 *(300 / \mathrm{T})^{4.4} \\
\mathrm{~K}_{i}=1.40 \mathrm{e}-12 *(300 / \mathrm{T})^{0.7}\end{array}$ \\
\hline (30) $\mathrm{N}_{2} \mathrm{O}_{5}+\mathrm{M} \rightarrow \mathrm{NO}_{2}+\mathrm{NO}_{3}+\mathrm{M}$ & $\mathrm{K}_{29} * 3.333 \mathrm{e} 26 * \exp (-10991 / \mathrm{T})$ \\
\hline (31) $\mathrm{NO}_{2}+\mathrm{OH}+\mathrm{M} \rightarrow \mathrm{HNO}_{3}+\mathrm{M}$ & $\begin{array}{l}\text { troe: } \mathrm{k}_{O}=2.40 \mathrm{e}-30 *(300 / \mathrm{T})^{3.1} \\
\mathrm{~K}_{i}=1.70 \mathrm{e}-11 *(300 / \mathrm{T})^{2.1}\end{array}$ \\
\hline (32) $\mathrm{HNO}_{3}+\mathrm{OH} \rightarrow \mathrm{NO}_{3}+\mathrm{H}_{2} \mathrm{O}$ & $\begin{array}{l}\operatorname{aux} 2+2.4 \mathrm{e}-14 * \exp (460 . / \mathrm{T}) \\
\operatorname{aux} 1=6.5 \mathrm{e}-34 * \exp (1335 / \mathrm{T}) * \operatorname{am} \\
\operatorname{aux} 2=\operatorname{aux} 1 /\left(1+\operatorname{aux} 1 *(2.7 \mathrm{e}-17 * \exp (2199 / \mathrm{T}))^{-1}\right)\end{array}$ \\
\hline (33) $\mathrm{NO}_{3}+\mathrm{NO} \rightarrow 2 * \mathrm{NO}_{2}$ & $1.50 \mathrm{e}-11 * \exp (170 / \mathrm{T})$ \\
\hline (34) $\mathrm{NO}_{3}+\mathrm{O} \rightarrow \mathrm{NO}_{2}+\mathrm{O}_{2}$ & $1.00 \mathrm{e}-11$ \\
\hline (35) $\mathrm{NO}_{3}+\mathrm{OH} \rightarrow \mathrm{HO}_{2}+\mathrm{NO}_{2}$ & $2.20 \mathrm{e}-11$ \\
\hline (36) $\mathrm{NO}_{3}+\mathrm{HO}_{2} \rightarrow \mathrm{OH}+\mathrm{NO}_{2}+\mathrm{O}_{2}$ & $3.50 \mathrm{e}-12$ \\
\hline (37) $\mathrm{NO}_{2}+\mathrm{HO}_{2}+\mathrm{M} \rightarrow \mathrm{HO}_{2} \mathrm{NO}_{2}+\mathrm{M}$ & $\begin{array}{l}\text { troe: } \mathrm{k}_{O}=1.80 \mathrm{e}-31 *(300 / \mathrm{T})^{3.2} \\
\mathrm{k}_{i}=4.70 \mathrm{e}-12 *(300 / \mathrm{T})^{1.4}\end{array}$ \\
\hline (38) $\mathrm{HO}_{2} \mathrm{NO}_{2}+\mathrm{OH} \rightarrow \mathrm{H}_{2} \mathrm{O}+\mathrm{NO}_{2}+\mathrm{O}_{2}$ & $1.30 \mathrm{e}-12 * \exp (380 / \mathrm{T})$ \\
\hline (39) $\mathrm{HO}_{2} \mathrm{NO}_{2}+\mathrm{M} \rightarrow \mathrm{HO}_{2}+\mathrm{NO}_{2}+\mathrm{M}$ & $\mathrm{k}_{37} * \exp (-10900 / \mathrm{T}) / 2.1 \mathrm{e}-27$ \\
\hline (40) $\mathrm{CH} 4+\mathrm{OH} \rightarrow \mathrm{CH}_{3} \mathrm{O}_{2}+\mathrm{H}_{2} \mathrm{O}$ & $2.45 \mathrm{e}-12 * \exp (-1775 / \mathrm{T})$ \\
\hline (41) $\mathrm{CH}_{3} \mathrm{O}_{2}+\mathrm{NO} \rightarrow \mathrm{CH}_{2} \mathrm{O}+\mathrm{NO}_{2}+\mathrm{HO}_{2}$ & $4.20 \mathrm{e}-12 * \exp (180 / \mathrm{T})$ \\
\hline (42) $\mathrm{CH}_{3} \mathrm{O}_{2}+\mathrm{HO}_{2} \rightarrow \mathrm{CH}_{3} \mathrm{OOH}+\mathrm{O}_{2}$ & $3.80 \mathrm{e}-13 * \exp (800 / \mathrm{T})$ \\
\hline (43) $\mathrm{CH}_{3} \mathrm{OOH}+\mathrm{OH} \rightarrow \mathrm{CH}_{3} \mathrm{O}_{2}+\mathrm{H}_{2} \mathrm{O}$ & $3.80 \mathrm{e}-12 * \exp (200 / \mathrm{T})$ \\
\hline (44) $\mathrm{CH}_{2} \mathrm{O}+\mathrm{NO}_{3} \rightarrow \mathrm{CO}+\mathrm{HO}_{2}+\mathrm{HNO}_{3}$ & $6.00 \mathrm{e}-13 * \exp (-2058 / \mathrm{T})$ \\
\hline (45) $\mathrm{CH}_{2} \mathrm{O}+\mathrm{OH} \rightarrow \mathrm{CO}+\mathrm{H}_{2} \mathrm{O}+\mathrm{H}$ & $1.00 \mathrm{e}-11$ \\
\hline (46) $\mathrm{CH}_{2} \mathrm{O}+\mathrm{O} \rightarrow \mathrm{HO}_{2}+\mathrm{OH}+\mathrm{CO}$ & $3.40 \mathrm{e}-11 * \exp (-1600 / \mathrm{T})$ \\
\hline (47) $\mathrm{CO}+\mathrm{OH} \rightarrow \mathrm{CO}_{2}+\mathrm{H}$ & $1.5 \mathrm{e}-13 *\left(1 .+6 . \mathrm{e}-7 * \mathrm{k}_{B} * \mathrm{am} * \mathrm{~T}\right)$ \\
\hline (48) $\mathrm{H}+\mathrm{O}_{2}+\mathrm{M} \rightarrow \mathrm{HO}_{2}+\mathrm{M}$ & $\begin{array}{l}\text { troe: } \mathrm{k}_{o}=5.70 \mathrm{e}-32 *(300 / \mathrm{T})^{1.6} \\
\mathrm{k}_{i}=7.50 \mathrm{e}-11\end{array}$ \\
\hline (49) $\mathrm{H}+\mathrm{O}_{3} \rightarrow \mathrm{OH}+\mathrm{O}_{2}$ & $1.40 \mathrm{e}-10 * \exp (-470 / \mathrm{T})$ \\
\hline
\end{tabular}


TABLE 2. (Continued)

\begin{tabular}{|c|c|}
\hline Reaction & $\begin{array}{c}\text { Rate coefficient } \\
\text { (from MOZART3 chemical module) }\end{array}$ \\
\hline (50) $\mathrm{H}+\mathrm{HO}_{2} \rightarrow 2 * \mathrm{OH}$ & $7.05 \mathrm{e}-11$ \\
\hline (51) $\mathrm{H}+\mathrm{HO}_{2} \rightarrow \mathrm{H}_{2}+\mathrm{O}_{2}$ & $7.29 \mathrm{e}-12$ \\
\hline (52) $\mathrm{H}+\mathrm{HO}_{2} \rightarrow \mathrm{H}_{2} \mathrm{O}+\mathrm{O}$ & $1.62 \mathrm{e}-12$ \\
\hline (53) $\mathrm{OH}+\mathrm{O} \rightarrow \mathrm{H}+\mathrm{O}_{2}$ & $2.20 \mathrm{e}-11 * \exp (120 / \mathrm{T})$ \\
\hline (54) $\mathrm{OH}+\mathrm{O}_{3} \rightarrow \mathrm{HO}_{2}+\mathrm{O}_{2}$ & $1.50 \mathrm{e}-12 * \exp (-880 / \mathrm{T})$ \\
\hline (55) $\mathrm{OH}+\mathrm{HO}_{2} \rightarrow \mathrm{H}_{2} \mathrm{O}+\mathrm{O}_{2}$ & $4.80 \mathrm{e}-11 * \exp (250 / \mathrm{T})$ \\
\hline (56) $\mathrm{OH}+\mathrm{OH} \rightarrow \mathrm{H}_{2} \mathrm{O}+\mathrm{O}$ & $4.20 \mathrm{e}-12 * \exp (-240 / \mathrm{T})$ \\
\hline (57) $\mathrm{OH}+\mathrm{OH}+\mathrm{M} \rightarrow \mathrm{H}_{2} \mathrm{O}_{2}+\mathrm{M}$ & troe: $\mathrm{k}_{o}=6.20 \mathrm{e}-31 *(300 / \mathrm{T})$ \\
\hline (58) $\mathrm{OH}+\mathrm{H}_{2} \rightarrow \mathrm{H}_{2} \mathrm{O}+\mathrm{H}$ & $\mathrm{k}_{i}=2.60 \mathrm{e}-11$ \\
\hline (58) $\mathrm{OH}+\mathrm{H}_{2} \rightarrow \mathrm{H}_{2} \mathrm{O}+\mathrm{H}$ & $5.50 \mathrm{e}-12 * \exp (-2000 / \mathrm{T})$ \\
\hline (59) $\mathrm{OH}+\mathrm{H}_{2} \mathrm{O}_{2} \rightarrow \mathrm{H}_{2} \mathrm{O}+\mathrm{HO}_{2}$ & $2.90 \mathrm{e}-12 * \exp (-160 / \mathrm{T})$ \\
\hline (60) $\mathrm{HO}_{2}+\mathrm{O} \rightarrow \mathrm{OH}+\mathrm{O}_{2}$ & $3.00 \mathrm{e}-11 * \exp (200 / \mathrm{T})$ \\
\hline (61) $\mathrm{HO}_{2}+\mathrm{O}_{3} \rightarrow \mathrm{OH}+2 * \mathrm{O}_{2}$ & $2.00 \mathrm{e}-14 * \exp (-680 / \mathrm{T})$ \\
\hline (62) $\mathrm{HO}_{2}+\mathrm{HO}_{2} \rightarrow \mathrm{H}_{2} \mathrm{O}_{2}+\mathrm{O}_{2}$ & $(2.3 e-13 * \exp (600 / T)+$ aux 1$) * \operatorname{aux} 2$ \\
\hline & aux $1=1.7 \mathrm{e}-33 *$ am $* \exp (1000 / \mathrm{T})$ \\
\hline & aux $2=1 .+1.4 \mathrm{e}-21 *$ am $* \mathrm{H}_{2} \mathrm{O} * \exp (2200 / \mathrm{T})$ \\
\hline (63) $\mathrm{H}_{2} \mathrm{O}_{2}+\mathrm{O} \rightarrow \mathrm{OH}+\mathrm{HO}_{2}$ & $1.40 \mathrm{e}-12 * \exp (-2000 / \mathrm{T})$ \\
\hline (64) $\mathrm{Cl}+\mathrm{O}_{3} \rightarrow \mathrm{ClO}+\mathrm{O}_{2}$ & $2.30 \mathrm{e}-11 * \exp (-200 / \mathrm{T})$ \\
\hline (65) $\mathrm{Cl}+\mathrm{H}_{2} \rightarrow \mathrm{HCl}+\mathrm{H}$ & $3.70 \mathrm{e}-11 * \exp (-2300 / \mathrm{T})$ \\
\hline (66) $\mathrm{Cl}+\mathrm{H}_{2} \mathrm{O}_{2} \rightarrow \mathrm{HCl}+\mathrm{HO}_{2}$ & $1.10 \mathrm{e}-11 * \exp (-980 / \mathrm{T})$ \\
\hline (67) $\mathrm{Cl}+\mathrm{HO}_{2} \rightarrow \mathrm{HCl}+\mathrm{O}_{2}$ & $1.80 \mathrm{e}-11 * \exp (170 / \mathrm{T})$ \\
\hline (68) $\mathrm{Cl}+\mathrm{HO}_{2} \rightarrow \mathrm{OH}+\mathrm{ClO}$ & $4.10 \mathrm{e}-11 * \exp (-450 / \mathrm{T})$ \\
\hline (69) $\mathrm{Cl}+\mathrm{CH}_{2} \mathrm{O} \rightarrow \mathrm{HCl}+\mathrm{HO}_{2}+\mathrm{CO}$ & $8.10 \mathrm{e}-11 * \exp (-30 / \mathrm{T})$ \\
\hline (70) $\mathrm{Cl}+\mathrm{CH} 4 \rightarrow \mathrm{CH}_{3} \mathrm{O}_{2}+\mathrm{HCl}$ & $9.60 \mathrm{e}-12 * \exp (-1360 / \mathrm{T})$ \\
\hline (71) $\mathrm{ClO}+\mathrm{O} \rightarrow \mathrm{Cl}+\mathrm{O}_{2}$ & $3.00 \mathrm{e}-11 * \exp (70 / \mathrm{T})$ \\
\hline (72) $\mathrm{ClO}+\mathrm{OH} \rightarrow \mathrm{Cl}+\mathrm{HO}_{2}$ & $7.40 \mathrm{e}-12 * \exp (270 / \mathrm{T})$ \\
\hline (73) $\mathrm{ClO}+\mathrm{OH} \rightarrow \mathrm{HCl}+\mathrm{O}_{2}$ & $3.20 \mathrm{e}-13 * \exp (320 / \mathrm{T})$ \\
\hline (74) $\mathrm{ClO}+\mathrm{HO}_{2} \rightarrow \mathrm{O}_{2}+\mathrm{HOCl}$ & $4.80 \mathrm{e}-13 * \exp (700 / \mathrm{T})$ \\
\hline (75) $\mathrm{ClO}+\mathrm{NO} \rightarrow \mathrm{NO}_{2}+\mathrm{Cl}$ & $6.40 \mathrm{e}-12 * \exp (290 / \mathrm{T})$ \\
\hline (76) $\mathrm{ClO}+\mathrm{NO}_{2}+\mathrm{M} \rightarrow \mathrm{ClONO}_{2}+\mathrm{M}$ & troe: $\mathrm{k}_{o}=1.80 \mathrm{e}-31 *(300 / \mathrm{T})^{3.4}$ \\
\hline & $\mathrm{k}_{i}=1.50 \mathrm{e}-11 *(300 / \mathrm{T})^{1.9}$ \\
\hline (77) $\mathrm{ClO}+\mathrm{ClO} \rightarrow 2 * \mathrm{Cl}+\mathrm{O}_{2}$ & $3.00 \mathrm{e}-11 * \exp (-2450 / \mathrm{T})$ \\
\hline (78) $\mathrm{ClO}+\mathrm{ClO} \rightarrow \mathrm{Cl}_{2}+\mathrm{O}_{2}$ & $1.00 \mathrm{e}-12 * \exp (-1590 / \mathrm{T})$ \\
\hline (79) $\mathrm{ClO}+\mathrm{ClO} \rightarrow \mathrm{Cl}+\mathrm{OClO}$ & $3.50 \mathrm{e}-13 * \exp (-1370 / \mathrm{T})$ \\
\hline (80) $\mathrm{ClO}+\mathrm{ClO}+\mathrm{M} \rightarrow \mathrm{Cl}_{2} \mathrm{O}_{2}+\mathrm{M}$ & troe: $\mathrm{k}_{o}=2.20 \mathrm{e}-32 *(300 / \mathrm{T})^{3.1}$ \\
\hline & $\mathrm{k}_{i}=3.50 \mathrm{e}-12 *(300 / \mathrm{T})$ \\
\hline (81) $\mathrm{Cl}_{2} \mathrm{O}_{2}+\mathrm{M} \rightarrow \mathrm{ClO}+\mathrm{ClO}+\mathrm{M}$ & $\mathrm{k}_{80} /(1.3 \mathrm{e}-27 * \exp (8744 / \mathrm{T}))$ \\
\hline (82) $\mathrm{HCl}+\mathrm{OH} \rightarrow \mathrm{H}_{2} \mathrm{O}+\mathrm{Cl}$ & $2.60 \mathrm{e}-12 * \exp (-350 / \mathrm{T})$ \\
\hline (83) $\mathrm{HCl}+\mathrm{O} \rightarrow \mathrm{Cl}+\mathrm{OH}$ & $1.00 \mathrm{e}-11 * \exp (-3300 / \mathrm{T})$ \\
\hline (84) $\mathrm{HOCl}+\mathrm{O} \rightarrow \mathrm{ClO}+\mathrm{OH}$ & $1.70 \mathrm{e}-13$ \\
\hline (85) $\mathrm{HOCl}+\mathrm{Cl} \rightarrow \mathrm{HCl}+\mathrm{ClO}$ & $2.50 \mathrm{e}-12 * \exp (-130 / \mathrm{T})$ \\
\hline (86) $\mathrm{HOCl}+\mathrm{OH} \rightarrow \mathrm{H}_{2} \mathrm{O}+\mathrm{ClO}$ & $3.00 \mathrm{e}-12 * \exp (-500 / \mathrm{T})$ \\
\hline (87) $\mathrm{ClONO}_{2}+\mathrm{O} \rightarrow \mathrm{ClO}+\mathrm{NO}_{3}$ & $2.90 \mathrm{e}-12 * \exp (-800 / \mathrm{T})$ \\
\hline (88) $\mathrm{ClONO}_{2}+\mathrm{OH} \rightarrow \mathrm{HOCl}+\mathrm{NO}_{3}$ & $1.20 \mathrm{e}-12 * \exp (-333 / \mathrm{T})$ \\
\hline (89) $\mathrm{ClONO}_{2}+\mathrm{Cl} \rightarrow \mathrm{Cl}_{2}+\mathrm{NO}_{3}$ & $6.50 \mathrm{e}-12 * \exp (-135 / \mathrm{T})$ \\
\hline (90) $\mathrm{Br}+\mathrm{O}_{3} \rightarrow \mathrm{BrO}+\mathrm{O}_{2}$ & $1.70 \mathrm{e}-11 * \exp (-800 / \mathrm{T})$ \\
\hline (91) $\mathrm{Br}+\mathrm{HO}_{2} \rightarrow \mathrm{HBr}+\mathrm{O}_{2}$ & $1.50 \mathrm{e}-11 * \exp (-600 / \mathrm{T})$ \\
\hline (92) $\mathrm{Br}+\mathrm{CH}_{2} \mathrm{O} \rightarrow \mathrm{HBr}+\mathrm{HO}_{2}+\mathrm{CO}$ & $1.70 \mathrm{e}-11 * \exp (-800 / \mathrm{T})$ \\
\hline (93) $\mathrm{BrO}+\mathrm{O} \rightarrow \mathrm{Br}+\mathrm{O}_{2}$ & $1.90 \mathrm{e}-11 * \exp (230 / \mathrm{T})$ \\
\hline (94) $\mathrm{BrO}+\mathrm{OH} \rightarrow \mathrm{Br}+\mathrm{HO}_{2}$ & $7.50 \mathrm{e}-11$ \\
\hline (95) $\mathrm{BrO}+\mathrm{HO}_{2} \rightarrow \mathrm{HOBr}+\mathrm{O}_{2}$ & $3.40 \mathrm{e}-12 * \exp (540 / \mathrm{T})$ \\
\hline (96) $\mathrm{BrO}+\mathrm{NO} \rightarrow \mathrm{Br}+\mathrm{NO}_{2}$ & $8.80 \mathrm{e}-12 * \exp (260 / \mathrm{T})$ \\
\hline (97) $\mathrm{BrO}+\mathrm{NO}_{2}+\mathrm{M} \rightarrow \mathrm{BrONO}_{2}+\mathrm{M}$ & troe: $\mathrm{k}_{o}=5.20 \mathrm{e}-31 *(300 / \mathrm{T})^{3.2}$ \\
\hline & $\mathrm{k}_{i}=6.90 \mathrm{e}-12 *(300 / \mathrm{T})^{2.9}$ \\
\hline (98) $\mathrm{BrO}+\mathrm{ClO} \rightarrow \mathrm{Br}+\mathrm{OClO}$ & $9.50 \mathrm{e}-13 * \exp (550 / \mathrm{T})$ \\
\hline (99) $\mathrm{BrO}+\mathrm{ClO} \rightarrow \mathrm{Br}+\mathrm{Cl}+\mathrm{O}_{2}$ & $2.30 \mathrm{e}-12 * \exp (260 / \mathrm{T})$ \\
\hline (100) $\mathrm{BrO}+\mathrm{ClO} \rightarrow \mathrm{BrCl}+\mathrm{O}_{2}$ & $4.10 \mathrm{e}-13 * \exp (290 / \mathrm{T})$ \\
\hline (101) $\mathrm{BrO}+\mathrm{BrO} \rightarrow 2 * \mathrm{Br}+\mathrm{O}_{2}$ & $1.50 \mathrm{e}-12 * \exp (230 / \mathrm{T})$ \\
\hline
\end{tabular}


TABLE 2. (Continued)

\begin{tabular}{ll}
\hline \hline \multicolumn{1}{c}{ Reaction } & \multicolumn{1}{c}{$\begin{array}{c}\text { Rate coefficient } \\
\text { (from MOZART3 chemical module) }\end{array}$} \\
\hline$(102) \mathrm{HBr}+\mathrm{OH} \rightarrow \mathrm{Br}+\mathrm{H}_{2} \mathrm{O}$ & $1.10 \mathrm{e}-11$ \\
$(103) \mathrm{CH}_{3} \mathrm{Cl}+\mathrm{Cl} \rightarrow \mathrm{HO}_{2}+\mathrm{CO}+2 * \mathrm{HCl}$ & $3.20 \mathrm{e}-11 * \exp (-1250 / \mathrm{T})$ \\
$(104) \mathrm{CH}_{3} \mathrm{Cl}+\mathrm{OH} \rightarrow \mathrm{Cl}+\mathrm{H}_{2} \mathrm{O}+\mathrm{HO}_{2}$ & $4.00 \mathrm{e}-12 * \exp (-1400 / \mathrm{T})$ \\
$(105) \mathrm{CH}_{3} \mathrm{CCl}+\mathrm{OH} \rightarrow \mathrm{H}_{2} \mathrm{O}+3.0 * \mathrm{Cl}$ & $1.80 \mathrm{e}-12 * \exp (-1550 / \mathrm{T})$ \\
$(106) \mathrm{HCF}_{2} \mathrm{C} 22+\mathrm{OH} \rightarrow \mathrm{Cl}+\mathrm{H}_{2} \mathrm{O}+\mathrm{CF}_{2} 2 \mathrm{O}$ & $1.00 \mathrm{e}-12 * \exp (-1600 / \mathrm{T})$ \\
$(107) \mathrm{CH}_{3} \mathrm{Br}+\mathrm{OH} \rightarrow \mathrm{Br}+\mathrm{H}_{2} \mathrm{O}+\mathrm{HO}_{2}$ & $4.00 \mathrm{e}-12 * \exp (-1470 / \mathrm{T})$ \\
\hline
\end{tabular}

(1994) with values of the solar F10.7 flux changing from 69 (mean for September 1986) to 235 (November 1989).

- Upper boundary concentrations for atomic hydrogen and oxygen are taken from the MSIS (Hedin 1991) climatology for the two values of the F10.7 flux mentioned above.

- The thermospheric NO production due to high energetic solar irradiance is assumed to increase by $30 \%$ for solar maximum conditions. This value has been chosen in order to reproduce reasonably the solar induced variation of thermospheric NO observed by SNOE (Barth et al. 2003). The auroral NO production does not differ between the two model runs.

To estimate the sensitivity of the model to doubling of $\mathrm{CO}_{2}$ we performed four simulations over $11 \mathrm{yr}$.

- $1 \times \mathrm{CO}_{2}$ : As the simulation for solar minimum, that is, with a $\mathrm{CO}_{2}$ mixing ratio of 360 ppmv fixed at the surface, but with the SW2 approach for solar heating (see section $2 \mathrm{a}$ ).

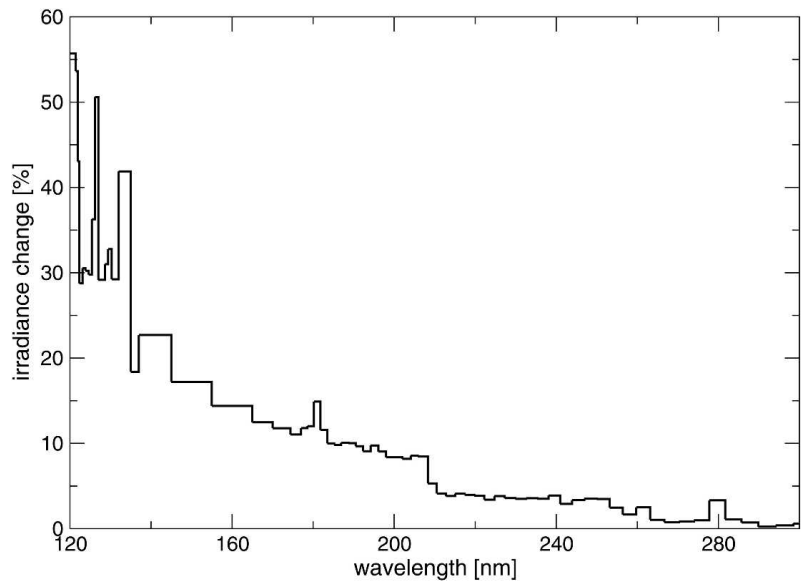

FIG. 2. Difference in the solar irradiance input between simulations for solar maximum and minimum with respect to solar minimum in percent. The step width indicates the wavelength resolution used in the computation of photodissociation and heating rates. Changes applied to wavelengths longer than $300 \mathrm{~nm}$ are in general smaller than $1 \%$.
- $2 \times \mathrm{CO}_{2}$ : As " $1 * \mathrm{CO}_{2}$ " but with a $\mathrm{CO}_{2}$ mixing ratio of 720 ppmv fixed at the surface, and the sea surface temperatures (SSTs) and sea ice modified according to a 720-ppmv $\mathrm{CO}_{2}$ climate (see below).

- $\operatorname{SST}\left(2 \times \mathrm{CO}_{2}\right)$ : As " $1 \times \mathrm{CO}_{2}$ " but with the SSTs and sea ice modified according to a 720-ppmv $\mathrm{CO}_{2}$ climate.

- $2 \times \mathrm{CO}_{2}$ (no SST): As " $1 \times \mathrm{CO}_{2}$ " but with a $\mathrm{CO}_{2}$ mixing ratio of 720 ppmv fixed at the surface.

To avoid transient changes in the 720-ppmv $\mathrm{CO}_{2}$ simulations, the initial values of the $\mathrm{CO}_{2}$ mixing ratio are doubled in the entire model domain. The first simulated year, however, is not used for the analysis of the runs. SSTs and sea ice cover for the 360-ppmv $\mathrm{CO}_{2}$ simulations are taken from the Atmospheric Model Intercomparison Project 2 (AMIP2) climatology. In the case of the 720-ppmv $\mathrm{CO}_{2}$ simulations, we added the differences of SSTs and sea ice cover from coupled simulations with the ECHAM5 atmospheric GCM (Roeckner et al. 2003) and the MPI Ocean Model (MPIOM; Marsland et al. 2003) for 720 and 360 ppmv of $\mathrm{CO}_{2}$, respectively. This results in an increase of July SSTs for doubled $\mathrm{CO}_{2}$ between about 0 and $1 \mathrm{~K}$ for high southern latitudes and 2 and $3 \mathrm{~K}$ in tropical and midlatitude northern oceans, and peaks of up to $6 \mathrm{~K}$ in single northern polar locations where the ice cover is also reduced significantly. The simulations " $\mathrm{SST}\left(2 \times \mathrm{CO}_{2}\right)$ " and " $2 \times \mathrm{CO}_{2}$ (no SST)" were performed to separate local radiative and nonlocal dynamical effects resulting from a doubling of the $\mathrm{CO}_{2}$ concentration.

All statistical significances for differences between simulations presented in this paper are computed using a Student's $t$ test.

The comparison of model results with satellite observations in the following section is done using the $20-y r$ solar minimum simulation. However, the differences between the reference simulations (solar minimum and $1 \times \mathrm{CO}_{2}$, respectively) that are due to the use of the slightly different approaches for solar heating (SW2 and SW1, respectively) are negligible for most parameters. 

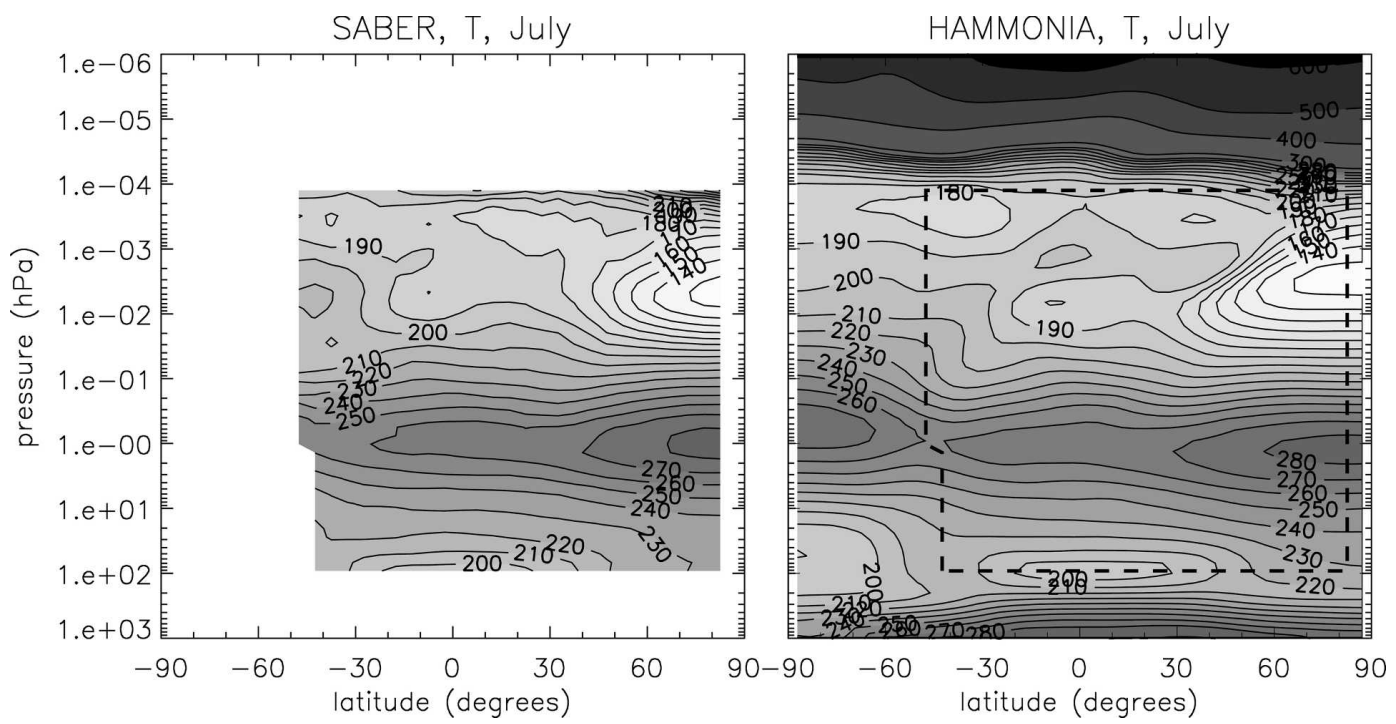

FIG. 3. Observed and simulated July temperatures (K). (left) Mean values for 1600 local time (LT) from SABER/TIMED observations between 1 and 15 July (mean of 2002 and 2005). (right) Simulated July mean values for $1600 \mathrm{LT}$. The dashed box marks the region of available observations.

The observational data used in the comparison are coming from the following sources: in the case of temperature and mesospheric ozone, version-1.06 data from the Sounding of the Atmosphere using Broadband Emission Radiometry (SABER) instrument (Mertens et al. 2001, 2004) on the Thermosphere, Ionosphere, Mesosphere Energetics and Dynamics (TIMED) satellite are used. SABER ozone mixing ratios shown here are those derived from observations of the $\mathrm{O}_{2}\left({ }^{1} \Delta\right)$ daytime emission at $1.27 \mu \mathrm{m}$. (The data are available online at http://saber.larc.nasa.gov.) In the case of zonal wind, methane, water vapor, and stratospheric ozone, the extended data from the Upper Atmosphere Research Satellite (UARS) Reference Atmosphere Project (URAP) in the version 1.0 are used. (All URAP data are taken from the Web site http://code916.gsfc. nasa.gov/Public/Analysis/UARS/urap/home.html.) Ozone data are described by Wang et al. (1996), and methane and water vapor are described by Randel et al. (1998). Wind data (Swinbank and Ortland 2003) are a composite of Met Office (MO) analyses below about $0.2 \mathrm{hPa}(\sim 60 \mathrm{~km})$ and UARS observations above. It should be noted, that the observations are mean values from limited periods: two years (2002 and 2005) in the case of SABER, and 4 (winds) or $7 \mathrm{yr}$ (chemical species) in the case of URAP.

\section{Comparison of model results with satellite observations}

In the following analysis of model results, we will focus on the month of July and on zonal mean values for most of the variables. Only in the case of temperature and ozone, is the comparison performed for a fixed local time because the observations are not uniformly distributed in local time and tidal variations are expected to be large in the mesopause region. All model results are 20-yr averages taken from the solar minimum simulation (see section 3). Most dynamical and chemical features presented in this section for the month of July are also simulated symmetrically in an at least a qualitatively very similar way for January.

Figure 3 shows temperatures from HAMMONIA and the SABER instrument as zonal means for 1600 local time. In general terms, the temperature distribution is similar in the model and the observations. Important features captured by the model include the cold summer mesopause and the warm winter stratopause (both caused mainly by the momentum deposition from gravity waves), different altitudes for summer and winter mesopause, the rapid increase in temperature in the lower thermosphere, the warm summer stratosphere, and the cold equatorial tropopause. The differences in the temperature at the equatorial mesopause region may be associated with phase shifts in tides. The temperature minimum below $180 \mathrm{~K}$ at about $30^{\circ}$ to $60^{\circ} \mathrm{S}$ and $10^{-4} \mathrm{hPa}$ is a feature of this particular local time and does not appear in the zonal mean temperature. The strong vertical wave pattern at the tropical mesopause is significantly weaker in the zonal mean than for 1600 LT. Simulated summer mesopause temperatures are about $10 \mathrm{~K}$ warmer in January than in July.

In the case of zonal winds, the model reproduces the 

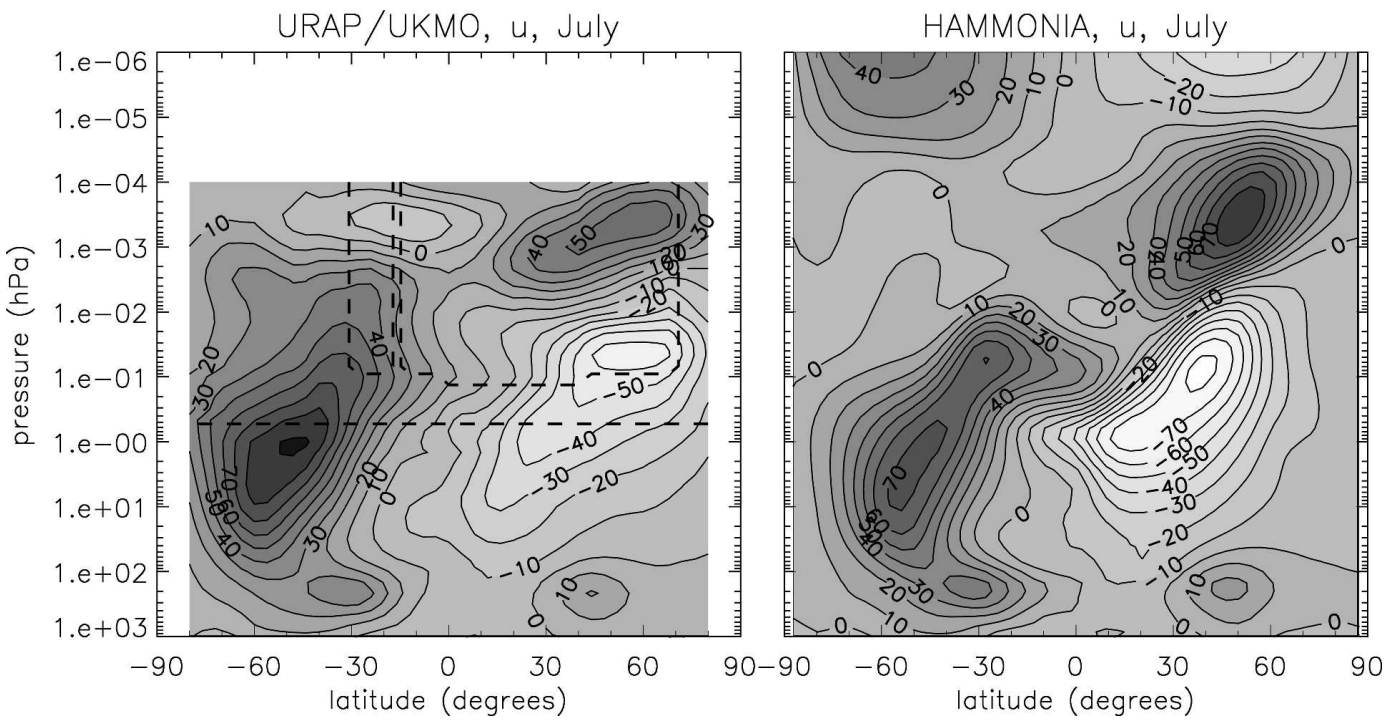

FIG. 4. Analyzed and simulated July zonal mean zonal winds $\left(\mathrm{m} \mathrm{s}^{-1}\right)$. (left) URAP analysis, which is composed of MO analyses below the horizontal dashed line and UARS observations within the two dashed boxes in the upper part. (right) Simulated values.

general pattern shown in the composite of $\mathrm{MO}$ analyses and observations from the High Resolution Doppler Imager (HRDI) on board the UARS (Swinbank and Ortland 2003) with some differences in the positions of the jets and in associated maximum speeds (Fig. 4). In particular, the reversal from strong easterly winds in the summer mesosphere to westerlies in the thermosphere is reasonably well reproduced. The winter westerly jet in the stratopause region is slightly weaker in the model than in the observations. In the Northern (summer) Hemisphere, the maxima of both the mesospheric easterly and the thermospheric westerly jets are stronger in the model than in the observations. The simulated mesospheric jet does not extend sufficiently poleward. To evaluate possible reasons for the differences it is useful to compare our simulations to those with the extended CMAM (Fomichev et al. 2002) where the mesospheric summer jet seems also to be underestimated at mid- to high latitudes. Given that the nonorographic gravity wave drag is a major forcing term at these altitudes and that both models use the same parameterization by Hines (1997a), this discrepancy between models and observations may be attributed to the treatment of gravity waves. Figure 5 shows a comparison of the annual cycle of monthly and zonal mean zonal winds from URAP and HAMMONIA close to the stratopause $(1 \mathrm{hPa}, \sim 50 \mathrm{~km})$ and in the mesopause region $(0.001 \mathrm{hPa}, \sim 92 \mathrm{~km})$. At both altitudes the simulated and observed annual cycles exhibit a qualitatively similar wind pattern in both hemispheres with easterlies in summer and westerlies in winter at the stratopause and with summertime westerly maxima at $0.001 \mathrm{hPa}$. At the stratopause, maximum wind speeds in the model are higher than in the URAP data and the jets do not extend sufficiently poleward. However, in both model and data, winds are weaker in the Northern than in the Southern Hemisphere. A difference in the mesopause region is the equatorial easterly winds of the URAP analysis, which are not featured in the model. These easterlies are supposed to be forced by the momentum deposition of the breaking diurnal tide (e.g., Lieberman and Hays 1994). An underestimation of the tidal forcing by the model may be a reason for this discrepancy. However, above and below $0.001 \mathrm{hPa}$ (not shown), the model simulates equatorial easterlies during nonsolstice conditions in a manner qualitatively similar to the URAP observations.

Zonal mean ozone mixing ratios for 1500 local time are compared to observations from SABER daytime data above $1 \mathrm{hPa}(\sim 50 \mathrm{~km})$ and the URAP climatological data below (Fig. 6). Again, the main observed features are well reproduced by the model: the primary and secondary ozone maxima, extending from pole to pole, respectively, in the stratosphere and near the mesopause, as well as the tertiary maximum (Marsh et al. 2001) in the high-latitude winter mesosphere. Up to the midmesosphere, observed and simulated mixing ratios agree well. The slight underestimation of ozone mixing ratios in the stratospheric maximum may be connected to the overestimation of water vapor (see below). The model mixing ratios in the secondary maximum are approximately $30 \%$ lower than the observations. The 

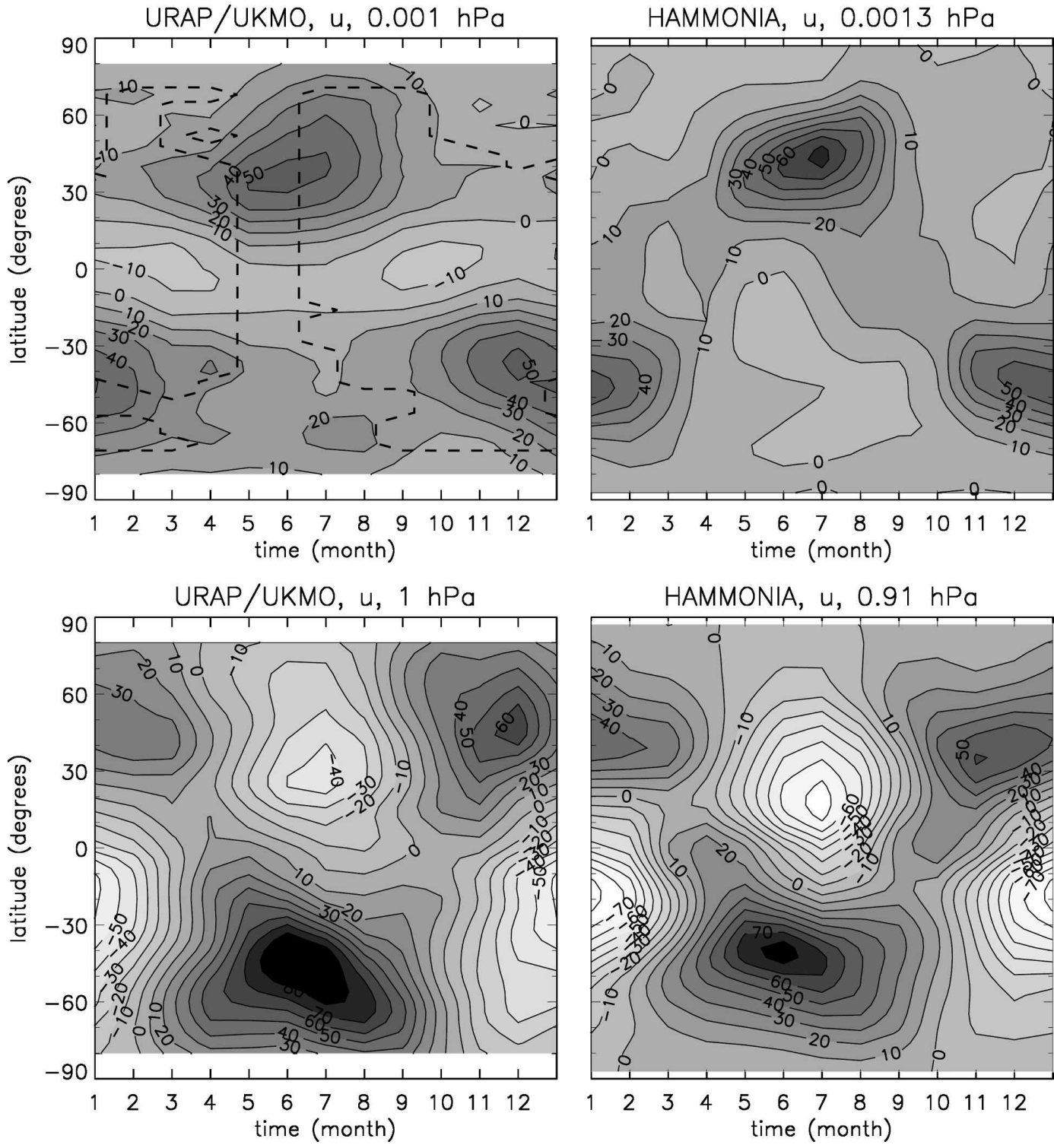

FIG. 5. Annual cycle of analyzed (left) URAP (see Fig. 4) and simulated (right) monthly and zonal mean zonal winds $\left(\mathrm{m} \mathrm{s}^{-1}\right.$ ) for pressure levels of about (bottom) 1 and (top) $0.001 \mathrm{hPa}$. In the regions poleward of the dashed lines and in the months of May and June at $0.001 \mathrm{hPa}$ the availability of observations is poor such that the URAP climatology is derived mainly by interpolation.

cause of this difference is unclear, but it should be noted that the new SABER observations have not been fully validated. However, the spatial patterns of the simulated and observed secondary daytime maxima are very similar. Both datasets show, for example, a remarkable local maximum in the highlatitude summer mesopause region. This feature is a consequence of very low temperatures (cf. Fig. 3) and can be explained from the ozone equation under photochemical equilibrium. Following Allen et al. (1984), in the upper mesosphere the ozone concentration is approximately given by

$$
\left[\mathrm{O}_{3}\right]=\frac{k_{\mathrm{O}+\mathrm{O}_{2}+M}[\mathrm{O}]\left[\mathrm{O}_{2}\right][M]}{J_{\mathrm{O}_{3}}+k_{\mathrm{H}+\mathrm{O}_{3}}[\mathrm{H}]+k_{\mathrm{O}+\mathrm{O}_{3}}[\mathrm{O}]},
$$

with the brackets indicating the number densities of chemical species, $k$ the reaction rate constants, $J_{\mathrm{O}_{3}}$ the photodissociation rate of ozone, and $M$ any reaction partner. Here $k_{\mathrm{O}+\mathrm{O}_{2}+M}$ is increasing exponentially with decreasing temperature. Additionally, any number density is, given a constant volume mixing ratio, inversely proportional to temperature. Therefore, ozone mixing ratios in the polar summer mesopause region are significantly higher than at lower latitudes although the 

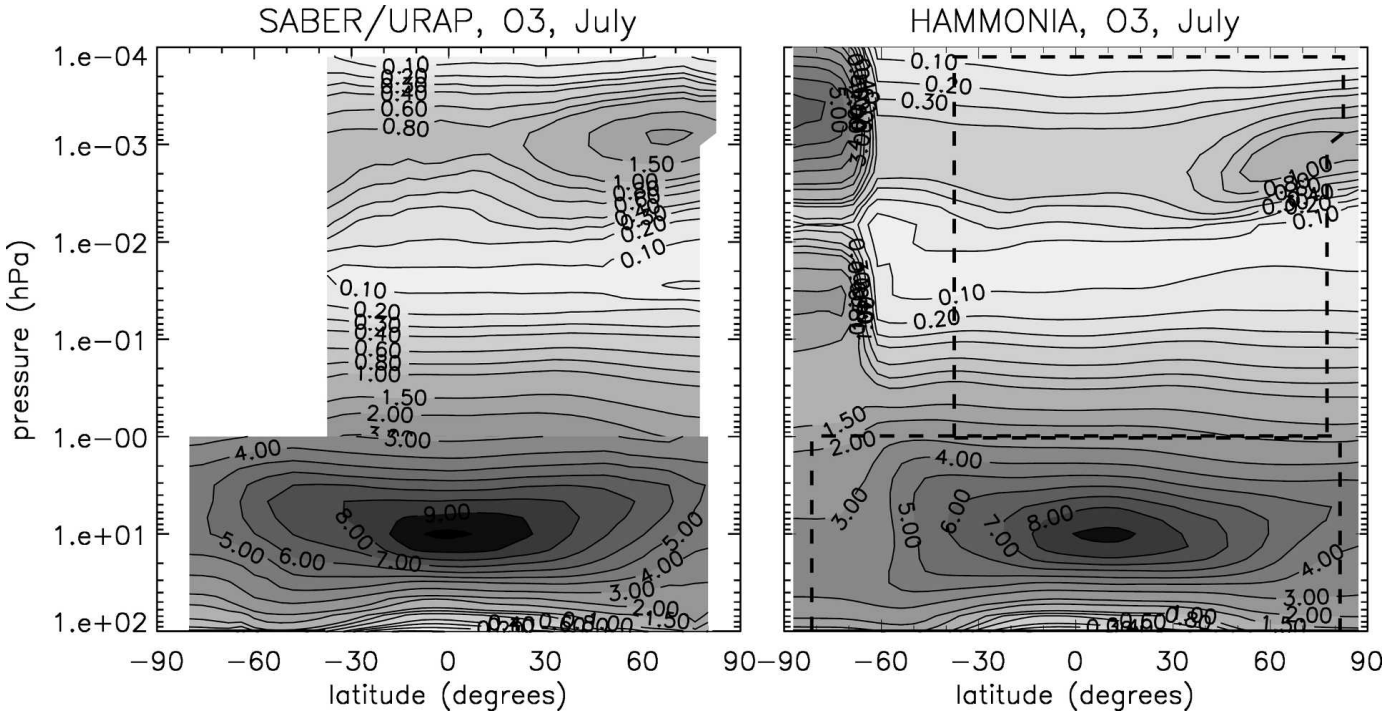

FIG. 6. Observed and simulated July daytime ozone mixing ratios (ppmv). (left) Mean values for 1500 LT from SABER observations between 1 and 15 July (mean of 2002 and 2005) above $1 \mathrm{hPa}$, and zonal mean July values from URAP below. (right) Simulated July mean values for 1500 LT. The dashed boxes mark the regions of available observations.

mixing ratio of atomic oxygen is relatively low (see Fig. 12) due to the polar upwelling.

Simulated nighttime ozone mixing ratios (not shown except for polar night values) in the secondary maximum are about one order of magnitude higher than those from daytime as also observed, for example, by the Cryogenic Infrared Spectrometers and Telescopes for the Atmosphere (CRISTA) satellite observations (Kaufmann et al. 2003).
The comparison of simulated mixing ratios for water vapor and methane with URAP data demonstrates the models' ability to simulate a realistic latitudinal and vertical distribution of important trace gases. In the case of water vapor (Fig. 7), the upward tilt of the water vapor isolines from the winter to the summer hemisphere is indicative of upwelling in the summer and downwelling in the winter (i.e., the Brewer-Dobson circulation). In addition, in the lower tropical stratosphere
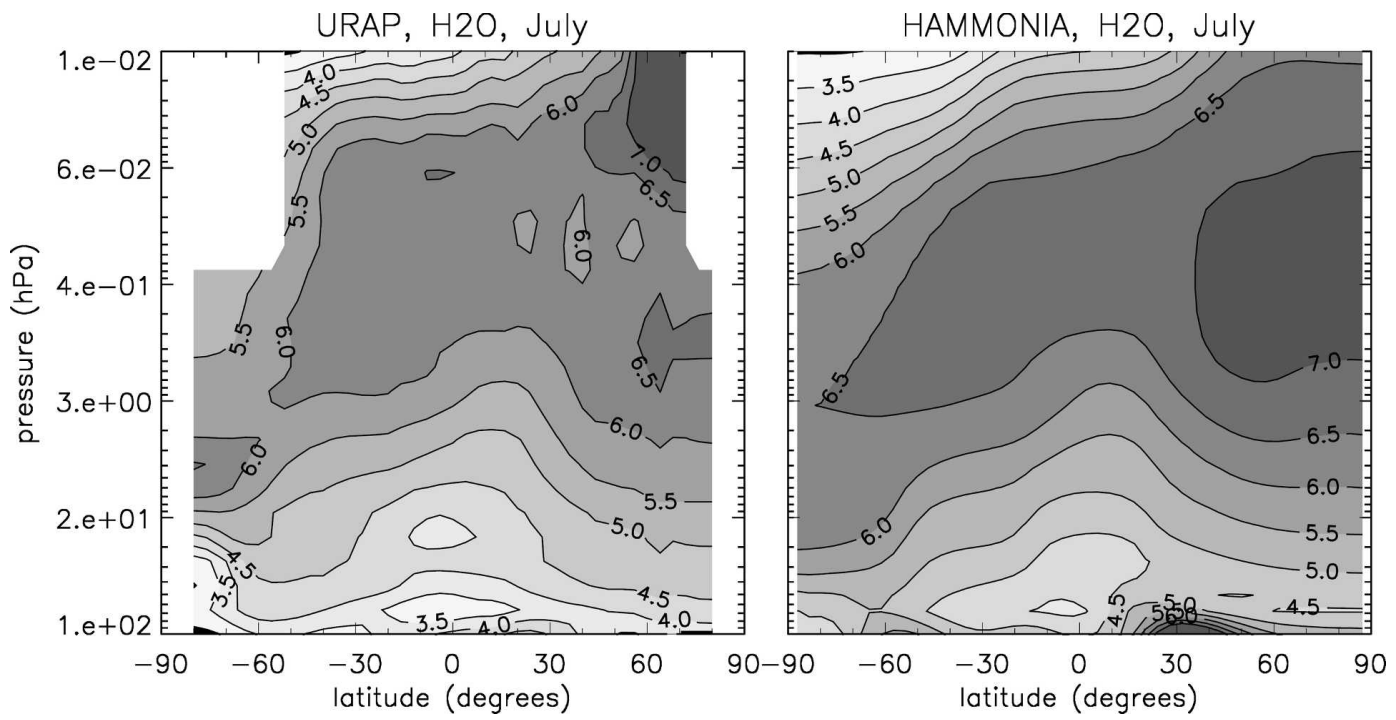

FIG. 7. Observed and simulated July zonal mean mixing ratios (ppmv) of water vapor. (left) Observations from URAP. (right) Simulated values. 


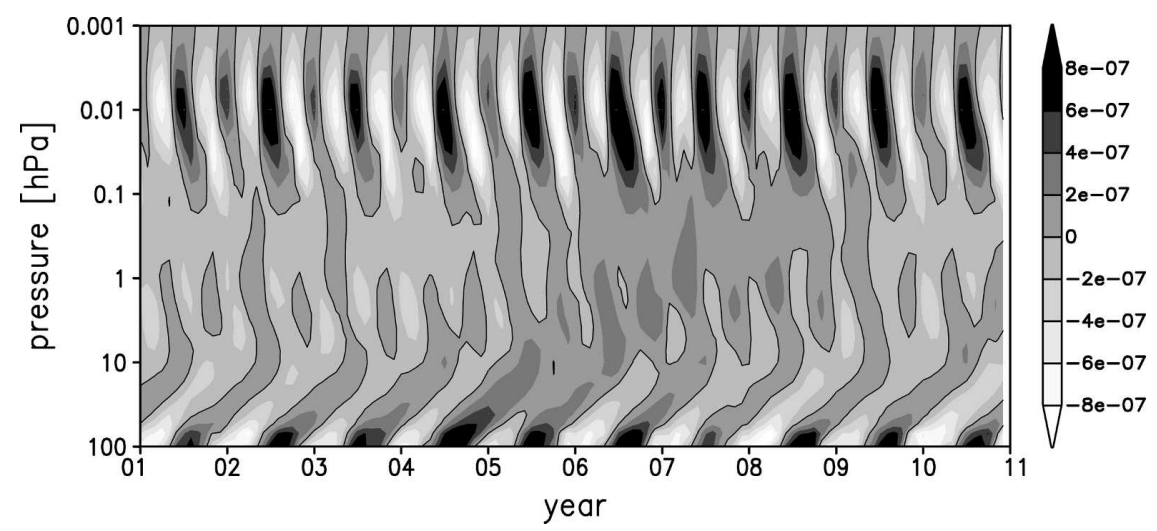

FIG. 8. Simulated deviation of the equatorial water vapor mixing ratio from the 10 -yr mean values for the respective altitudes. Mixing ratios are monthly and zonal mean values for $10 \mathrm{yr}$ of the solar minimum simulation. The isoline indicates the zero deviation.

close to the tropopause, the water vapor distribution in the model is asymmetric, with higher water vapor around $30^{\circ} \mathrm{N}$, in agreement with a monsoon source of moisture for the stratosphere (Gettelman et al. 2004). In most of the stratosphere and mesosphere, simulated water vapor mixing ratios are up to $1 \mathrm{ppm}$ higher than in the URAP data. The water vapor level in the model is very sensitive to the temperature in the equatorial tropopause region (see below). An error in this parameter and the relatively coarse vertical model resolution may lead to an overestimation of the water vapor transport across the tropopause. The high observed mixing ratios in the high-latitude upper mesosphere in summer may be a consequence of the downward transport and evaporation of icy particles from polar mesospheric clouds (von Zahn and Berger 2003), which are not included in our model. Figure 8 presents the time evolution over 10 simulated years of the equatorial water vapor mixing ratio in terms of its deviation from the 10 -yr mean value at heights from the surface to $10^{-3}$ $\mathrm{hPa}(\sim 92 \mathrm{~km})$. The model reproduces the well-known features like the "tape recorder" (Mote et al. 1996) in the lower stratosphere and the semiannual oscillation (SAO) close to the stratopause (Randel et al. 1998). The tape recorder is currently slightly too fast because of the missing quasi-biennial oscillation (QBO; see Giorgetta et al. 2006). An SAO with relatively large amplitude and almost opposite phase to the stratopause SAO is simulated for the mesopause region. This latter feature has been clearly observed in temperatures and zonal winds (Garcia et al. 1997) and is also detected in water vapor time series observed by the Halogen Occultation Experiment (HALOE) instrument as presented by Steil et al. (2003). An interesting feature is the positive tropopause water vapor anomaly in year 4 of Fig. 8 that is connected to a strong positive tempera- ture anomaly. This event is leading to enhanced water vapor mixing ratios in the middle atmosphere for several years.

In the case of methane (Fig. 9), the equatorial rate of vertical decrease is well captured by the model, indicating that upwelling is realistic. In addition, in the winter (southern) hemisphere, the methane isolines show a flattening at midlatitudes in the stratosphere, indicative of isentropic horizontal mixing. The Brewer-Dobson circulation manifests itself as a latitudinal gradient of methane (as for water vapor) in the mesosphere with lower values at the winter pole with respect to the summer pole.

\section{Sensitivity to the 11-yr solar cycle}

\section{a. Model results}

Figure 10 shows the solar cycle effect on the main components of the energy budget in the mesosphere and lower thermosphere: solar heating (including chemical heating), infrared cooling (including heating in the near-IR $\mathrm{CO}_{2}$ bands), and molecular heat conduction. The increased radiation for solar maximum conditions leads to a substantial increase in solar heating above approximately $0.01 \mathrm{hPa}(\sim 80 \mathrm{~km})$. Up to $10^{-4}$ $\mathrm{hPa}(\sim 105 \mathrm{~km})$ this increase amounts to about $2 \mathrm{~K}$ day $^{-1}$. In this region, about $60 \%$ of the increase (as well as of the absolute heating rate) is due to chemical heating. The increased heating is balanced to a large degree by enhanced infrared cooling. Above $10^{-4} \mathrm{hPa}$ the increase in solar heating becomes very large. In a small region around $5 \times 10^{-5} \mathrm{hPa}(\sim 110 \mathrm{~km})$ the major change of the energy budget is provided by heating due to molecular diffusion (conduction), which more than doubles. In the thermosphere, heat is transported downward by conduction, leading to a positive tem- 

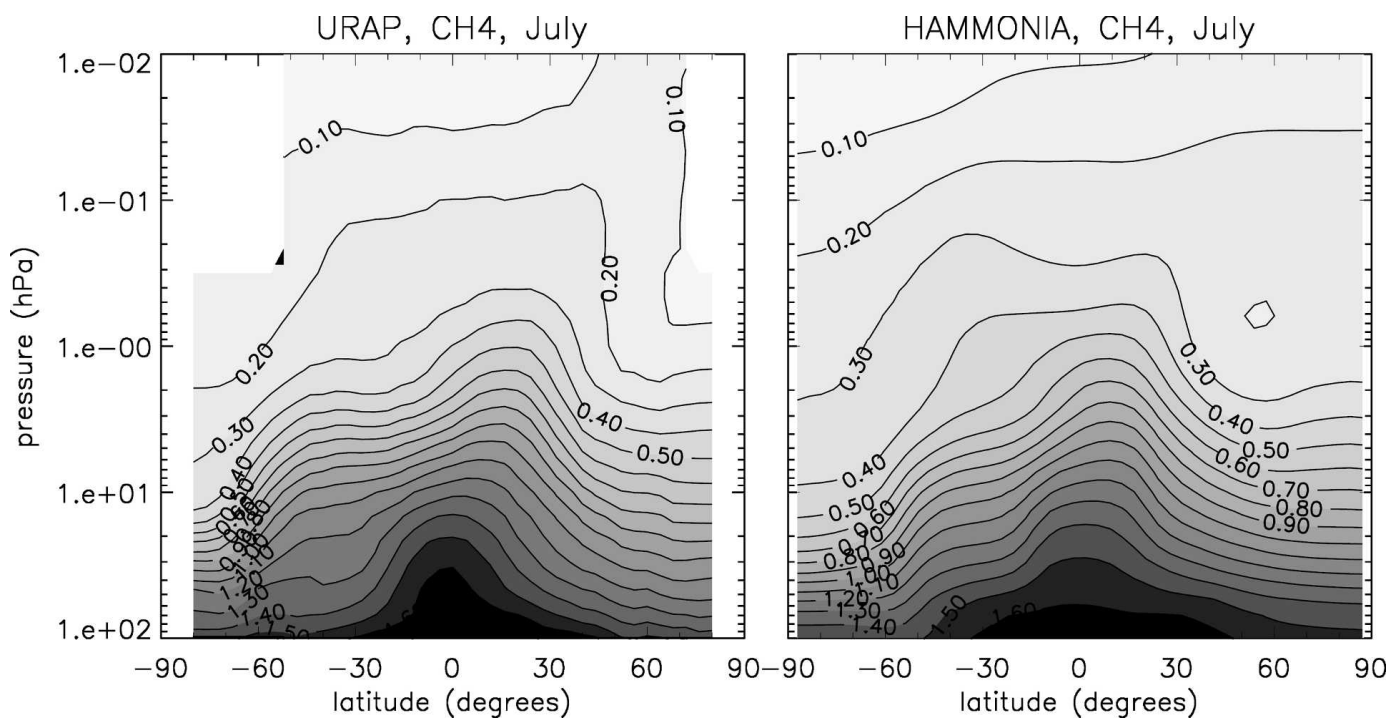

FIG. 9. Same as in Fig. 7, but for methane.

perature effect of conduction in this height range. Above $2 \times 10^{-5} \mathrm{hPa}(\sim 120 \mathrm{~km})$, the conduction effect becomes negative, and it is the main balancing process for solar heating and its increase.

This paper's focus is on the mesosphere and lower thermosphere. However, because dynamics of the upper atmosphere may be substantially influenced by nonlocal responses feeding back from the lower part of the atmosphere, we present in Fig. 11 the solar cycle effect on zonal mean temperature, zonal wind, and vertical residual wind from the surface to $10^{-5} \mathrm{hPa}(\sim 130 \mathrm{~km})$.

The July zonal mean temperature difference between solar maximum and minimum (Fig. 11) is positive everywhere above approximately the tropopause except for the Southern (winter) Hemisphere poleward of about $40^{\circ} \mathrm{S}$. Here, the signal is significantly positive only above about $0.03 \mathrm{hPa}(\sim 75 \mathrm{~km})$. The stratospheric temperature response (with values of about $1 \mathrm{~K}$ at the tropical stratopause) is well within the range of 0.4 to $2 \mathrm{~K}$ from different observational analyses, which are presented, for example, by Rozanov et al. (2004). In the mesopause region, the change lies between 2 and $10 \mathrm{~K}$, with values increasing in general with altitude and toward the summer pole. This latitudinal gradient is due to a change in the radiative budget. In high summer latitudes at around $0.001 \mathrm{hPa}$ the net radiative effect is a warming that is mainly due to the strong increase in chemical heating (which is an indirect radiative effect)
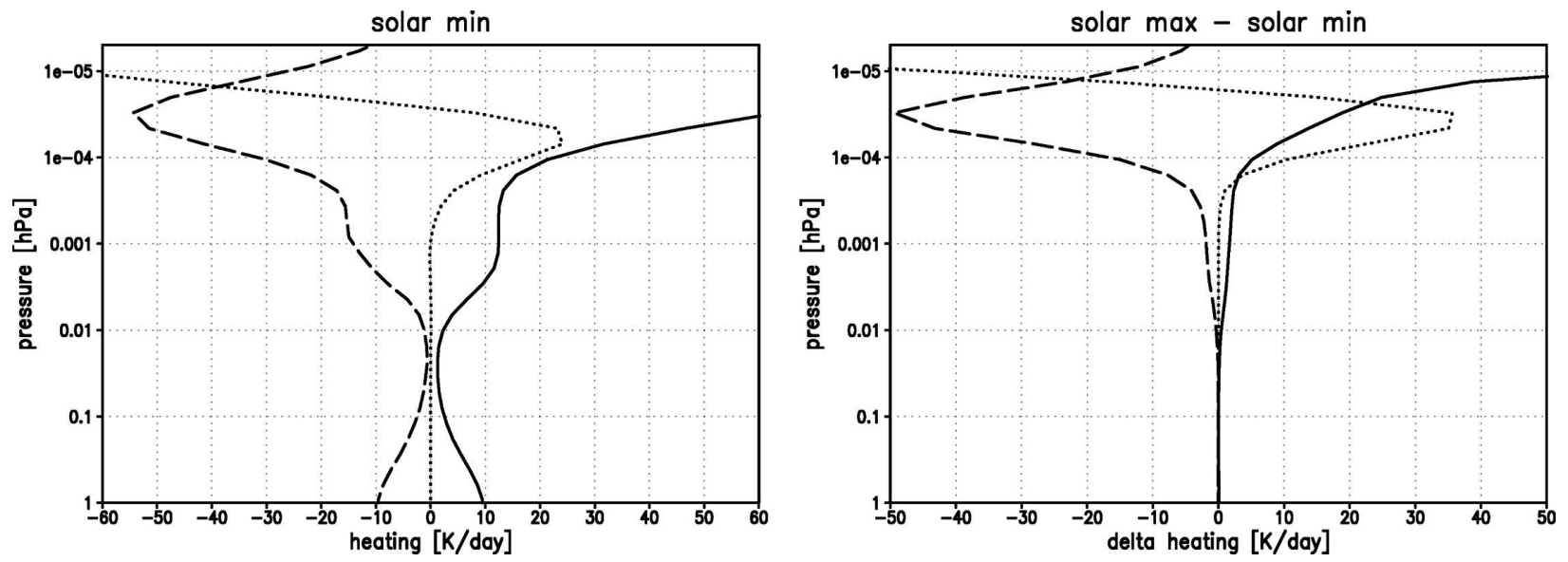

FIG. 10. Annual mean global average values for the major components of the atmospheric energy budget in $\mathrm{K}_{\text {day }}{ }^{-1}$. Solid lines denote solar heating including chemical heating, dashed lines denote infrared cooling/heating, and dotted lines denote molecular heat conduction. (left) Solar minimum simulation. (right) Changes for solar maximum with respect to the solar minimum simulation. 

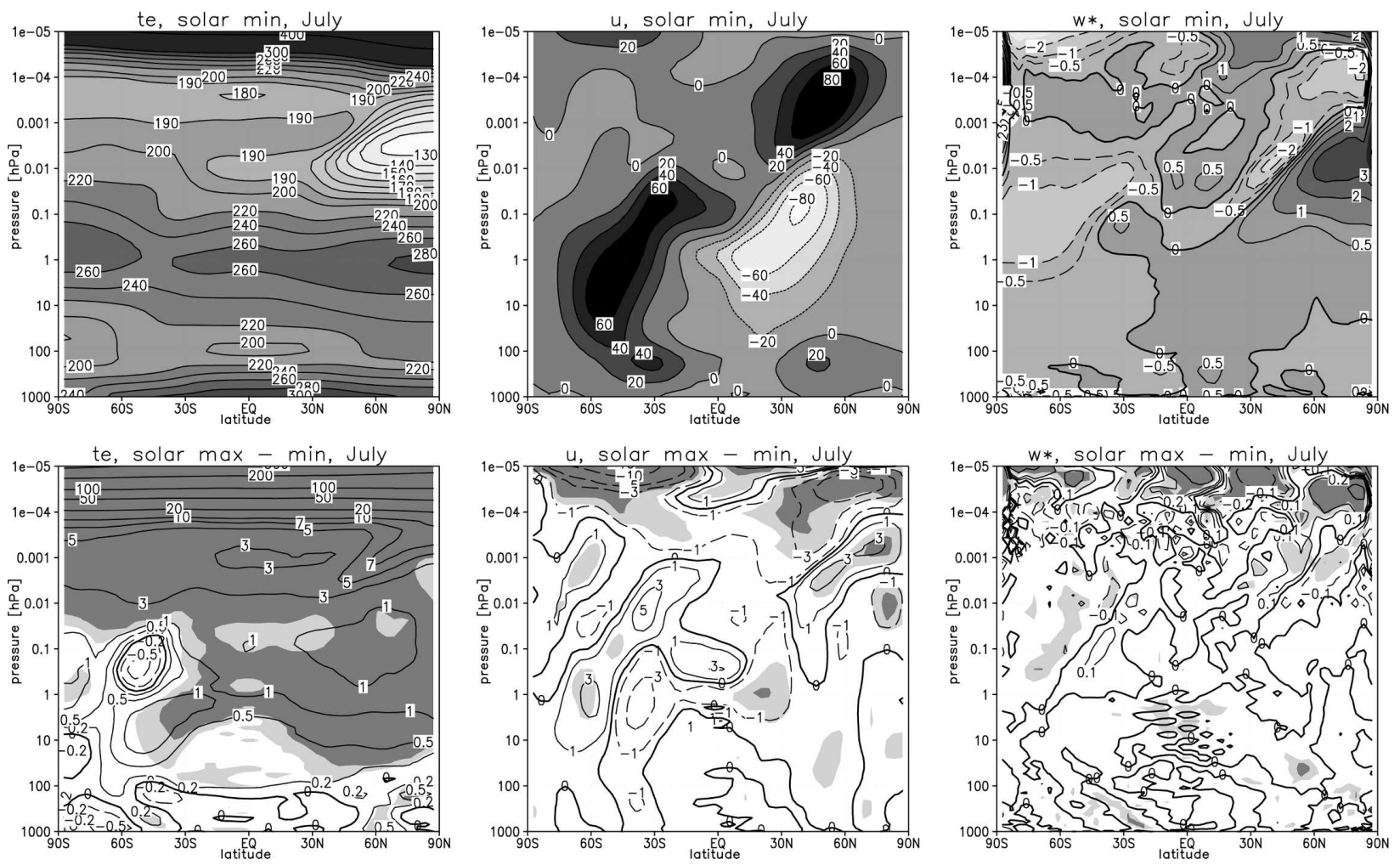

FIG. 11. Zonal mean values of temperature (K), zonal wind $\left(\mathrm{m} \mathrm{s}^{-1}\right)$, and the residual vertical wind $\left(\mathrm{cm} \mathrm{s}^{-1}\right)$ as simulated in the solar minimum run for July, and the changes for solar maximum with respect to the solar minimum run in the respective units. Statistical significance of the changes that is larger than $90 \%$ (99\%) is indicated by light (dark) gray shading.

caused by the increase in ozone (see below). The absolute minimum temperature at the summer mesopause changes only by about $1.5 \mathrm{~K}$. The larger changes are occurring above the mesopause. The general heating leads to an expansion of the atmosphere. Figure 1 shows an annual and global mean expansion of approximately $1 \mathrm{~km}$ at $10^{-4} \mathrm{hPa}(\sim 105 \mathrm{~km})$ and $100 \mathrm{~km}$ at the model top $\left(1.7 \times 10^{-7} \mathrm{hPa}\right)$.

Regarding the July zonal mean zonal wind difference, only relatively small changes can be seen in Fig. 11 except close to $10^{-5} \mathrm{hPa}(\sim 130 \mathrm{~km}$, and above; not shown). Here, the zonal wind is directly affected by a change in the ion drag, which is a result of the ion drag increasing with geopotential height and the expansion of the atmosphere. However, the increase of the ion drag for solar maximum is probably underestimated in these simulations as we use the same profile of the ion drag coefficient for solar maximum and minimum. Hong and Lindzen (1976) suggest larger coefficients for solar maximum. In the summer hemisphere, the change in the zonal wind implies a weakening of the lower thermospheric westerlies and the mesospheric easterlies. Such wind changes are consistent with reduced upwelling between 0.01 and $0.001 \mathrm{hPa}(\sim 80$ and $92 \mathrm{~km})$, and reduced downwelling above this layer. The zonal wind response can be explained with the thermal wind equation. The positive meridional gradient in the highlatitude temperature change in the mesopause region is causing a negative vertical gradient in the zonal wind. The solar response of the MLT region includes also changes in the gravity wave drag (not shown in the figures), but they are more likely a response to the changes in the wind field than their cause.

Figure 12 shows the solar cycle response in zonal mean July volume mixing ratios for ozone, atomic oxygen, nitric oxide, the hydroxyl radical, water vapor, and methane. Mesospheric ozone is characterized by a substantial diurnal variation with nighttime mixing ratios being about an order of magnitude larger than daytime values above $0.01 \mathrm{hPa}$. Therefore, considering zonal mean values does not seem to be adequate. However, as the relative solar responses of day- and nighttime ozone exhibit very similar patterns, we present zonal mean values also for this particular compound. In general, the photochemistry leading to ozone production and destruction is amplified for enhanced solar irradiance, which leads to a strengthening of the ozone maxima and an ozone decrease in regions with already 

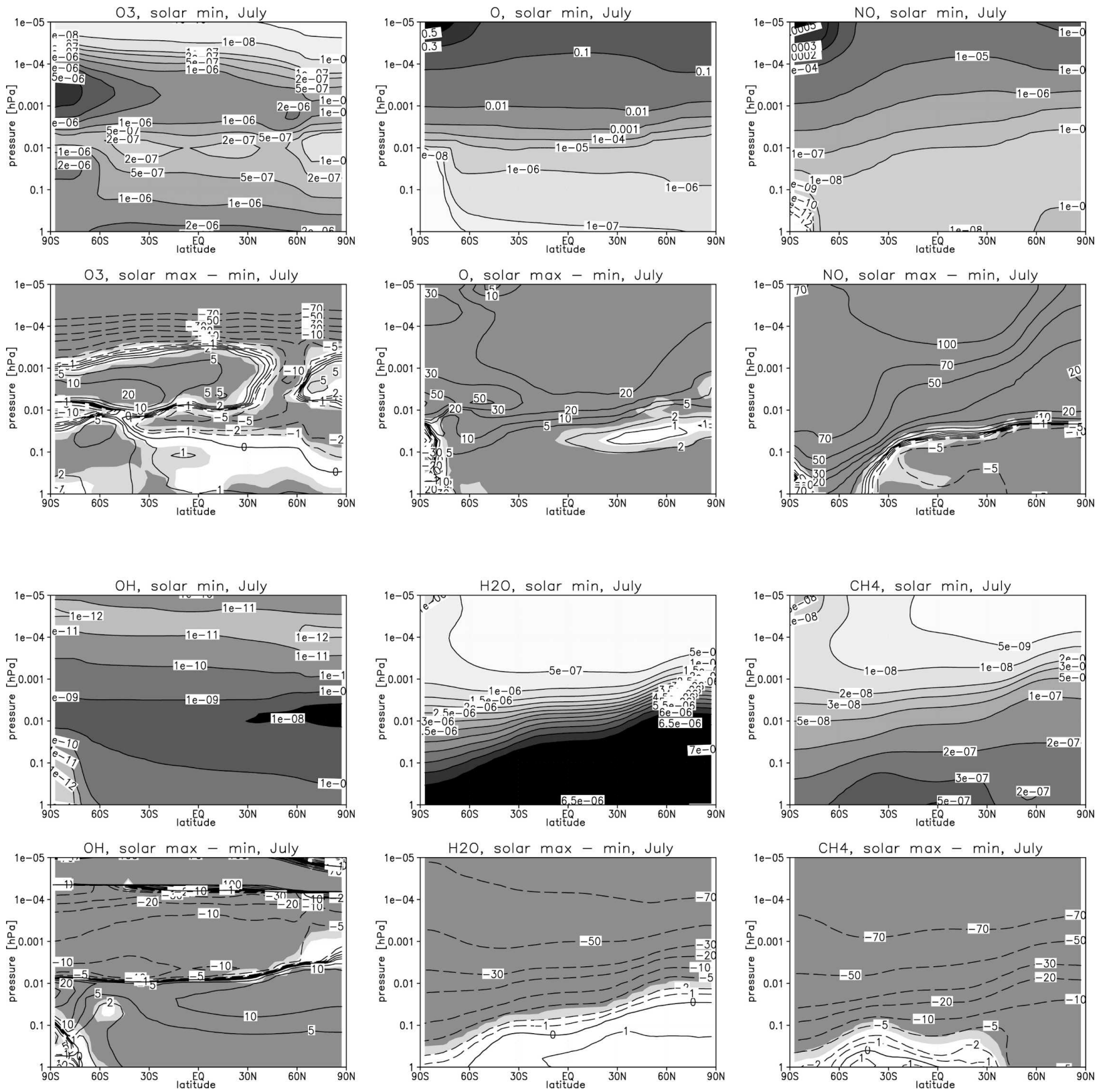

FIG. 12. Zonal mean July volume mixing ratios for $\mathrm{O}_{3}, \mathrm{O}, \mathrm{NO}, \mathrm{OH}, \mathrm{H}_{2} \mathrm{O}$, and $\mathrm{CH}_{4}$ as simulated in the solar minimum run, and the relative changes (\%) for solar maximum with respect to solar minimum. Statistical significance of the changes that is larger than $90 \%$ $(99 \%)$ is indicated by light (dark) gray shading.

low mixing ratios. The increase in the secondary maximum of up to $20 \%$ results from the enhanced mixing ratio of $\mathrm{O}$, which is a consequence of increased photodissociation of $\mathrm{O}_{2}$. The general decrease in a small height region below $0.01 \mathrm{hPa}$ is mainly due to the enhanced mixing ratio of $\mathrm{OH}$. For the relation of mesospheric ozone and atomic oxygen to odd hydrogen $\left(\mathrm{HO}_{x}=\mathrm{H}+\mathrm{OH}+\mathrm{HO}_{2}\right)$ see Brasseur and Offermann (1986) and Marsh et al. (2003). The higher temperature at solar maximum tends to decrease ozone. At the altitude of the secondary maximum at midnorthern latitudes, this decreasing effect is stronger than the ozone increase resulting from higher mixing ratios of atomic oxygen. The complicated structure of ozone response can also be seen in Fig. 13, which shows a horizontal cross section at $2 \times 10^{-3} \mathrm{hPa}(\sim 90 \mathrm{~km})$. The ozone response is significantly negative only at nighttime between approximately $45^{\circ}$ and $65^{\circ} \mathrm{N}$. This behavior can 

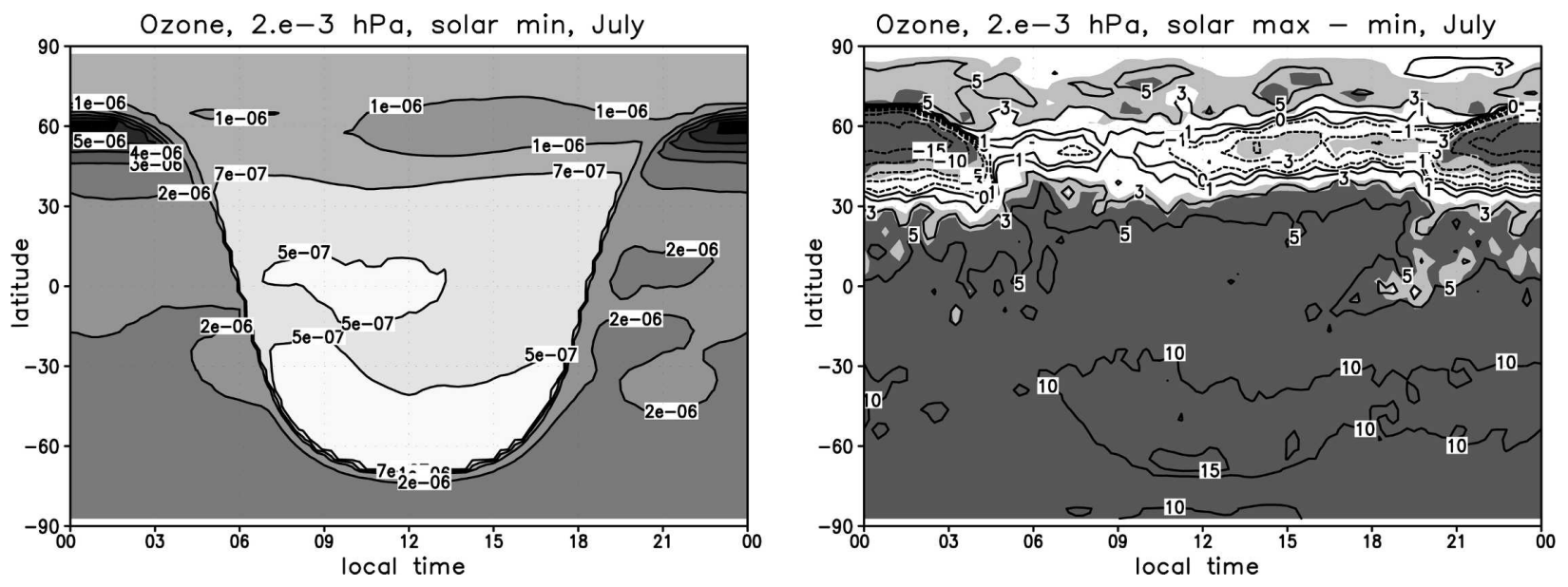

FIG. 13. July mean ozone volume mixing ratios at an altitude of $2 \times 10^{-3} \mathrm{hPa}(\sim 90 \mathrm{~km})$ for given latitudes and local times. (left) Solar minimum simulation. (right) Relative changes (\%) for solar maximum with respect to solar minimum. Statistical significance of the changes that is larger than $90 \%(99 \%)$ is indicated by light (dark) gray shading.

again be explained by the low summer temperatures (see section 4) and the nonlinearity of the temperature dependence of ozone. Figure 13 shows also that for most latitudes the changes in nighttime and daytime ozone are similar.

Zonal mean mixing ratios of $\mathrm{H}_{2} \mathrm{O}$ and $\mathrm{CH}_{4}$ exhibit a decrease for solar maximum, which is mainly an effect of increased photodissociation particularly by the approximately $60 \%$ increase of Lyman- $\alpha$ radiation. In the case of water vapor, the decrease is characterized by a strong latitudinal dependence and reaches down to about $1 \mathrm{hPa}(\sim 50 \mathrm{~km})$ in the winter hemisphere and only to about $0.03 \mathrm{hPa}(\sim 75 \mathrm{~km})$ in summer. This tilt is again the result of the prevailing circulation. The increase in the NO mixing ratio depends strongly on the assumption made for the increase in thermospheric NO production (see section 2e). However, less than half of the thermospheric NO increase can be explained by this imposed change of external production. The larger part of the change results from the large temperature changes that affect rate constants, and specifically the rate of the $\left(\mathrm{N}+\mathrm{O}_{2} \rightarrow \mathrm{NO}+\mathrm{O}\right)$ reaction [see, e.g., McEwan and Phillips (1975) for a description of NO chemistry]. The downwelling in the winter hemisphere leads to a significant $\mathrm{NO}_{x}$ increase down to the upper stratosphere, especially near the pole.

All dynamical and chemical features of the solar response described above for the month of July are also simulated symmetrically in a qualitatively very similar way for January.

\section{b. Comparison with other models}

A relatively large number of models have been applied to assess the effect of solar variability on tempera- ture, dynamics and chemistry of the stratosphere. See, for example, a recent study with a coupled threedimensional climate-chemistry model by Rozanov et al. (2004) and references therein. The annual mean stratospheric response of temperature (about $1 \mathrm{~K}$ at the equatorial stratopause) and ozone (about 3\% in the upper stratosphere) simulated with HAMMONIA lies well within the large variety of observational and modeling results listed by Rozanov et al. (2004).

For the mesosphere and lower thermosphere, the number of studies is small. Several studies addressed the effect of the 27-day rotational variation with onedimensional (Brasseur et al. 1987; Summers et al. 1990; Chen et al. 1997) or two-dimensional (Zhu et al. 2003) chemical dynamical models. The long-term (11-yr solar cycle) variability was studied with different versions of the National Center for Atmospheric Research (NCAR) Simulation of Chemistry, Radiation, and Transport of Environmentally Important Species (SOCRATES) interactive 2D model by Huang and Brasseur (1993, hereafter called HB1993) and Khosravi et al. (2002, hereafter called K2002). Most of their results can qualitatively be confirmed by our simulations although an accurate comparison of the studies is difficult because results are given for different seasons. In addition, the top of most models is located at a considerably lower altitude than in our case. While HB1993 simulate a temperature peak-to-peak response to solar activity in the mesopause region of about $10 \mathrm{~K}$, the value of about $5 \mathrm{~K}$ reported by K2002 is close to our simulations. As in our model, HB1993 detect only small changes in mesospheric winds. The statement of K2002 that dynamical feedbacks cause most of the change in temperature above $75 \mathrm{~km}(\sim 0.02 \mathrm{hPa})$ cannot be confirmed by our 


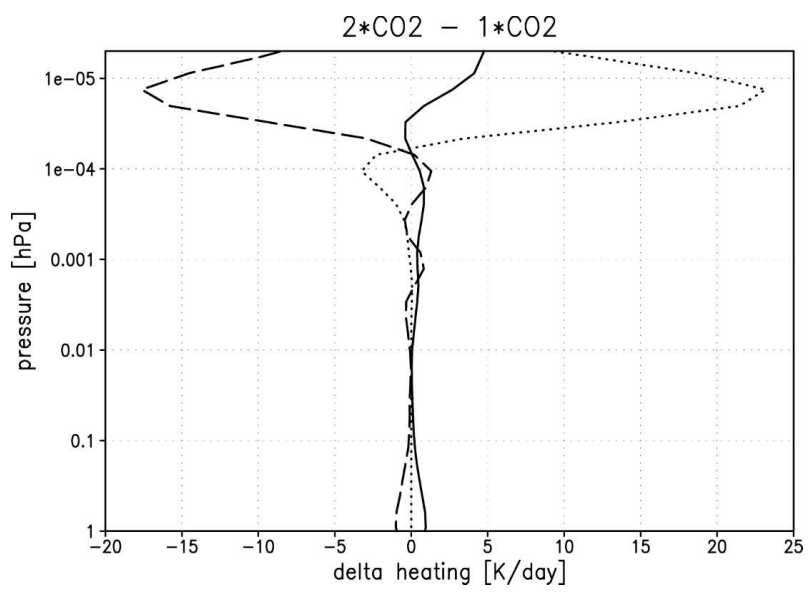

FIG. 14. Changes in the annual mean global average values for the major components of the atmospheric energy budget in $\mathrm{K}$ day $^{-1}$ for the $\times \mathrm{CO}_{2}$ simulation with respect to $1 \times \mathrm{CO}_{2}$. Solid lines denote solar heating including chemical heating, dashed lines denote infrared cooling/heating, and dotted lines denote molecular heat conduction.

model. In the case of ozone, the response patterns simulated by K2002 are relatively similar to the patterns simulated by HAMMONIA but with slightly higher amplitudes. HB1993 simulate a very large response in atomic oxygen and ozone mixing ratios in a small layer above $80 \mathrm{~km}(\sim 0.01 \mathrm{hPa})$ with increases of up to $120 \%$ and $55 \%$, respectively. Several other important features like the change in sign for the $\mathrm{OH}$ response around $0.01 \mathrm{hPa}$ and the strong $\mathrm{H}_{2} \mathrm{O}$ response reaching down below the stratopause in summer are simulated in all models.

\section{Sensitivity to $\mathrm{CO}_{2}$ doubling}

The cooling resulting from infrared $\mathrm{CO}_{2}$ emissions is a major contribution to the energy budget of the middle atmosphere and lower thermosphere. The rapid in- crease of the atmospheric $\mathrm{CO}_{2}$ concentration resulting from anthropogenic emissions is therefore expected to lead, in general, to a substantial cooling in this height range. Heating due to absorption of near-infrared radiation by $\mathrm{CO}_{2}$ can potentially counteract the cooling. However, according to Fomichev et al. (2004b) this effect is expected to be small. The simulation of the atmosphere for a doubled $\mathrm{CO}_{2}$ concentration with respect to present-day conditions has become a common benchmark experiment for climate models, and the respective simulation with HAMMONIA not only provides insight in the future atmosphere but also allows comparisons with a variety of similar studies.

\section{a. Model results}

Figure 14 shows the change in the main components of the energy budget in the mesosphere and lower thermosphere induced by a doubling in the atmospheric $\mathrm{CO}_{2}$ concentration. As can be expected, the infrared cooling (which is mainly due to infrared emission in the $15-\mu \mathrm{m} \mathrm{CO}_{2}$ band) increases strongly in the regions where this contribution is of importance: below $0.1 \mathrm{hPa}$ $(\sim 65 \mathrm{~km})$ and above $10^{-4} \mathrm{hPa}(\sim 105 \mathrm{~km})$. In the lower mesosphere this increase is partly balanced by increased solar heating as a consequence of the ozone feedback (see below). Between $10^{-4}$ and $10^{-5} \mathrm{hPa}$ $(\sim 105$ and $130 \mathrm{~km})$ the major balancing is achieved through an increase in the heating produced by conduction.

The $\mathrm{CO}_{2}$ doubling effect on zonal mean July temperature and winds is given in Fig. 15. As expected, the temperature decreases significantly almost everywhere above the tropopause. The general cooling leads to a shrinking of the atmosphere (Fig. 1) that reaches $1 \mathrm{~km}$ slightly above the stratopause and $10 \mathrm{~km}$ around $10^{-6}$ $\mathrm{hPa}(\sim 180 \mathrm{~km})$. While the decrease in the lower me-
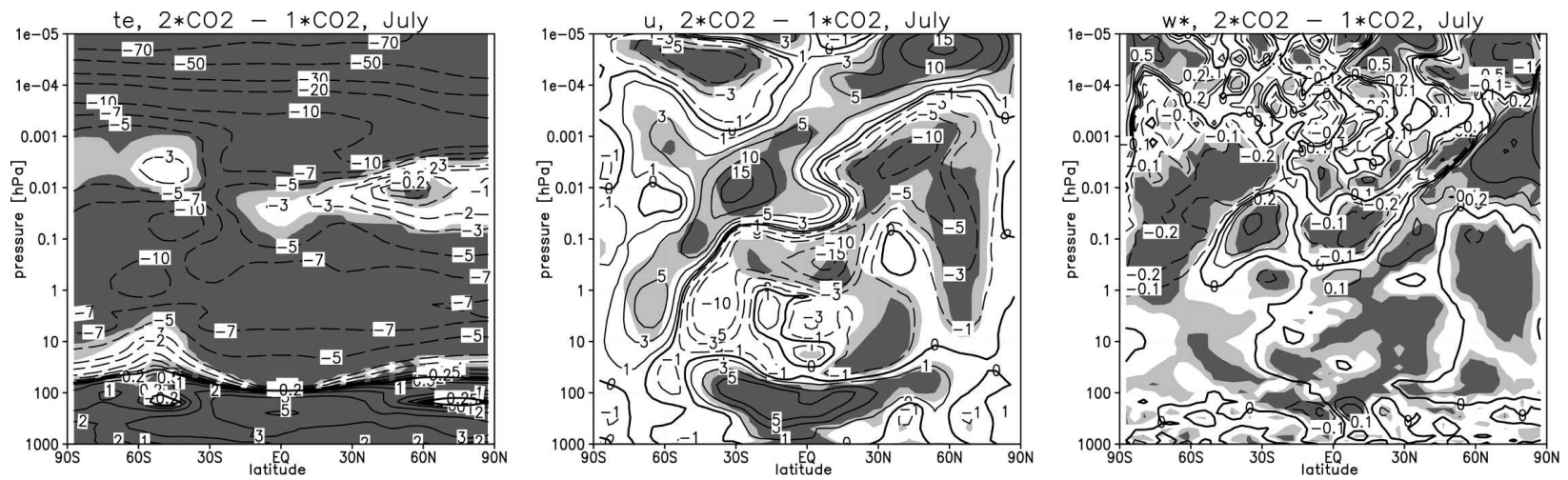

FIG. 15. Changes of July zonal mean temperature (K), zonal wind $\left(\mathrm{m} \mathrm{s}^{-1}\right)$, and the residual vertical wind $\left(\mathrm{cm} \mathrm{s}^{-1}\right)$ for the $2 \times \mathrm{CO}_{2}$ simulation with respect to $1 \times \mathrm{CO}_{2}$. Statistical significance of the changes that is larger than $90 \%(99 \%)$ is indicated by light (dark) gray shading. 

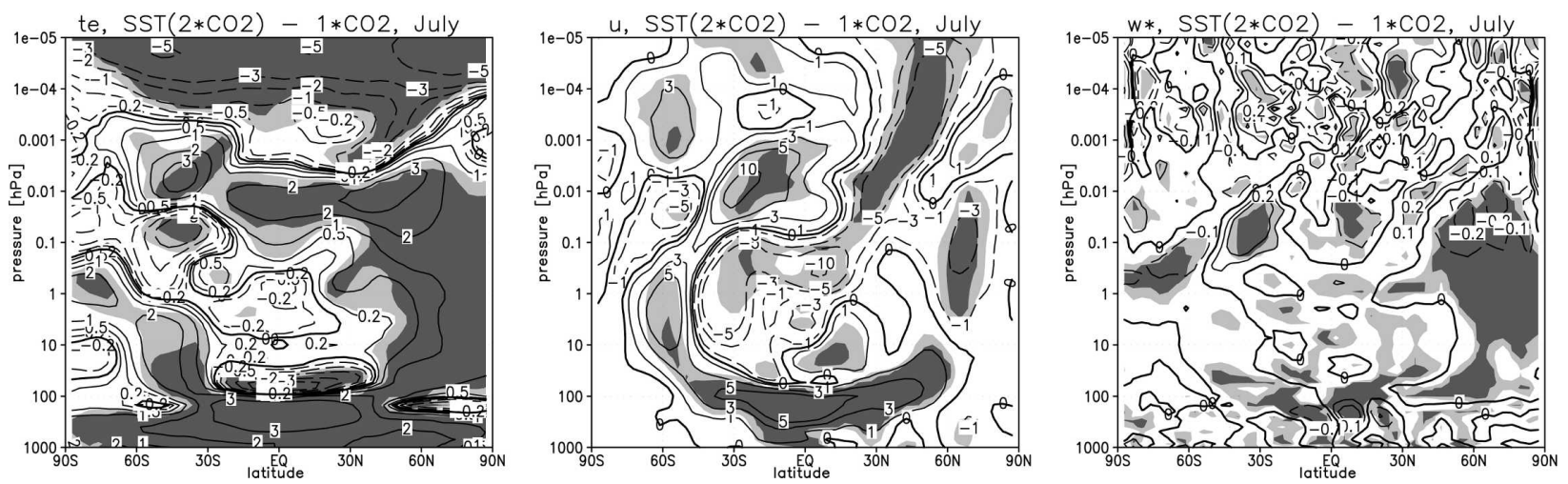

FIG. 16. Same as in Fig. 15, but for the $\operatorname{SST}\left(2 \times \mathrm{CO}_{2}\right)$ simulation with respect to $1 \times \mathrm{CO}_{2}$.

sosphere reaches $10 \mathrm{~K}$, the mesopause region shows the smallest, and in some places insignificant, cooling. This is the region where radiative transfer in the infrared $\mathrm{CO}_{2}$ bands provides very little cooling or can even heat the atmosphere due to the energy exchange with warmer underlying layers. In addition, in this region, the near-IR $\mathrm{CO}_{2}$ bands provide heating.

In the following, it will be shown that dynamical effects can also influence the temperature response in the middle and upper atmosphere and contribute to the small effect around the mesopause. In our simulation, the dynamical response in the MLT is characterized by a general increase in the residual circulation with an increased downwelling in the polar winter mesosphere and increased upwelling around the mesopause near the summer pole (Fig. 15). Consequently, zonal wind and temperature changes are less pronounced in the polar winter upper mesosphere, where the increased downwelling contributes to a warming and weaker zonal winds. Zonal wind and temperature changes are instead more pronounced above the summer polar mesopause region, where an upward shift of the summer easterlies and of the associated wind reversal, together with increased westerlies close to the $10^{-5} \mathrm{hPa}(\sim 130$ $\mathrm{km})$ level are predicted by the model. The analysis of two additional simulations $\left[\mathrm{SST}\left(2 \times \mathrm{CO}_{2}\right)\right.$, Fig. 16, with only the SSTs changed; and $2 \times \mathrm{CO}_{2}$ (no SST), Fig. 17, with only the $\mathrm{CO}_{2}$ mixing ratio increased] allows the separation of remote dynamical from local radiative effects of the $\mathrm{CO}_{2}$ doubling. Changes in the zonal and the residual vertical winds with respect to the reference simulation show large similarities between the $2 \times \mathrm{CO}_{2}$ and SST $(2 \times \mathrm{CO})$ simulations (cf. Figs. 15 and 16) at least up to the middle mesosphere while in the thermosphere the responses of the $2 \times \mathrm{CO}_{2}$ and the $2 \times \mathrm{CO}_{2}$ (no SST) experiments are similar (cf. Figs. 15 and 17). The sum of the two separate responses, for both temperature and winds, is almost equal to the changes observed in the combined simulation.

The positive temperature response to changing SSTs in the uppermost mesosphere suggests that the limited cooling in this region produced in response to a doubling of $\mathrm{CO}_{2}$ is partly caused by dynamics. Though it is difficult to separate causes and consequences of the complex dynamical interactions in the middle atmosphere, we suggest the following mechanism. The tem-
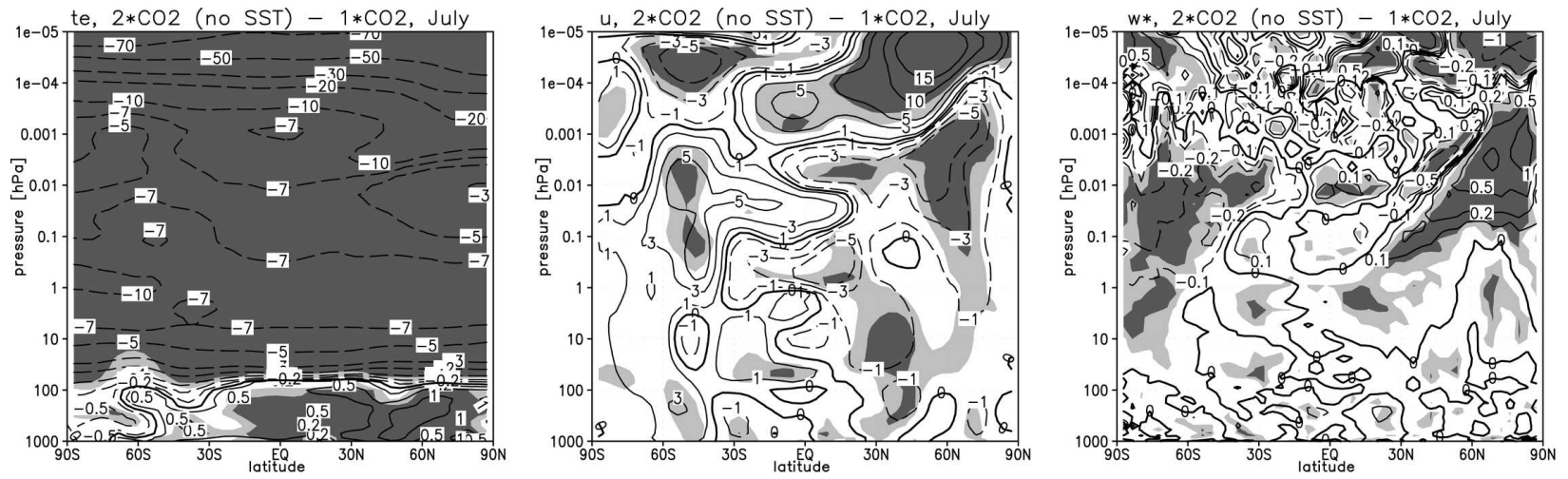

FIG. 17. Same as in Fig. 15, but for the $2 \times \mathrm{CO}_{2}$ (no SST) simulation with respect to $1 \times \mathrm{CO}_{2}$ (cf. Fig. 11). 

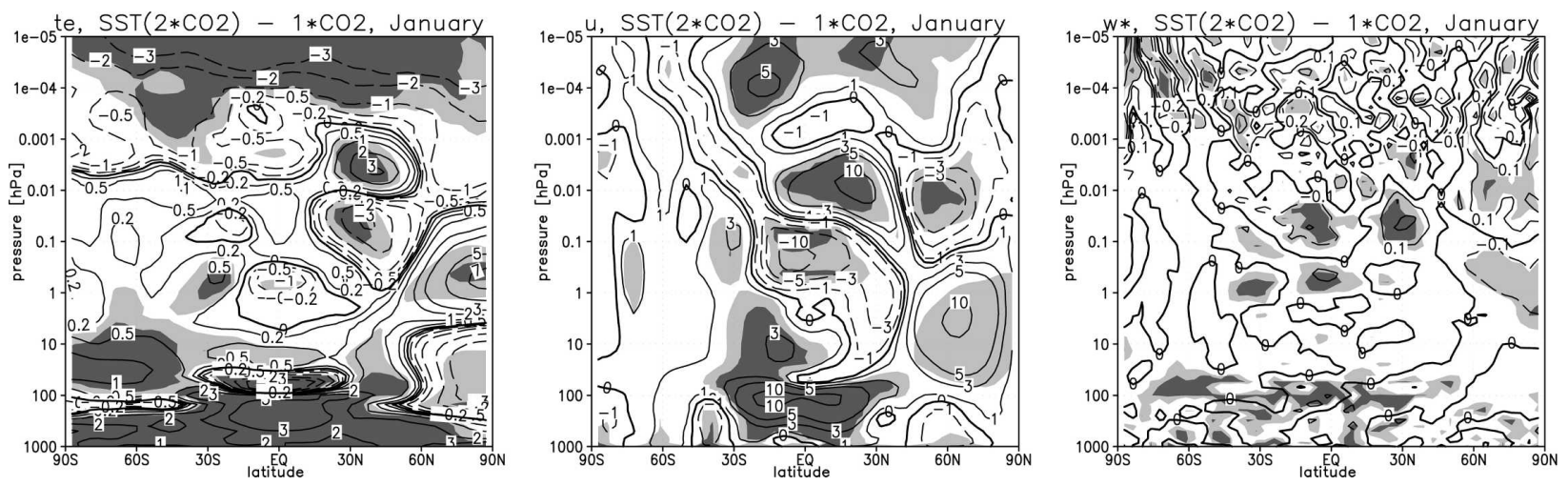

FIG. 18. Same as in Fig. 15, but for the $\operatorname{SST}\left(2 \times \mathrm{CO}_{2}\right)$ simulation with respect to $1 \times \mathrm{CO}_{2}$.

perature increase in the summer hemisphere in the $\operatorname{SST}\left(2 \times \mathrm{CO}_{2}\right)$ experiment is due to the decrease in upwelling leading to less adiabatic and advective cooling. The almost global positive temperature effect close to the mesopause is mainly due to a decrease in residual zonal southward winds between about 0.01 and 0.001 $\mathrm{hPa}$ leading to less advection of cold air. The reason for the decreased residual circulation at these altitudes is a reduction in the gravity wave forcing, which is mainly induced by changed propagation conditions with, in particular, the decrease of the wintertime westerly jet around $30^{\circ} \mathrm{S}$ at the stratopause. In the upper summer mesosphere, the decrease of upwelling in the $\operatorname{SST}\left(2 \times \mathrm{CO}_{2}\right)$ experiment is more than compensated for by the increase observed in the $2 \times \mathrm{CO}_{2}$ (no SST) experiment. The total effect of $\mathrm{CO}_{2}$ doubling in this height region is therefore an increase in residual circulation. The reason for the increased upwelling is the latitudinal gradient in the temperature response leading to a changing circulation via the thermal wind relation. In the very cold summer polar mesopause the longwave radiative effect of an increase in $\mathrm{CO}_{2}$ is, in contrast to most other regions in the atmosphere, a warming causing this gradient in the temperature response.

The upper mesospheric heating in the $\operatorname{SST}\left(2 \times \mathrm{CO}_{2}\right)$ experiment can be observed during Northern Hemisphere summer from June to September but has its maximum in July. In the southern summer however (see Fig. 18), an almost global warming of the mesopause region cannot be observed. Reduced upwelling is leading to a warming of the summer hemisphere. Changes in gravity wave forcing and subsequent mesopause meridional winds are much less significant than in July, so that the change in advective heating is small. The total $\mathrm{CO}_{2}$ doubling effect $\left(2 \times \mathrm{CO}_{2}\right.$ minus $1 \times \mathrm{CO}_{2}$; not shown) shows therefore a less pronounced mesopause cooling minimum in January than in July.

As described earlier, the $\mathrm{CO}_{2}$ mixing ratio is fixed at the surface but calculated in the atmosphere. This is why the actual mixing ratio is not exactly doubled at all altitudes. Figure 19 shows that the $\mathrm{CO}_{2}$ increase above $0.01 \mathrm{hPa}(\sim 80 \mathrm{~km})$ is larger than $100 \%$. This can partly be explained by a modification of the ratio between $\mathrm{CO}_{2}$ and $\mathrm{CO}$. The increase in $\mathrm{OH}$ (see below) increases this ratio via the reaction $\mathrm{CO}+\mathrm{OH} \rightarrow \mathrm{CO}_{2}+\mathrm{H}$. Additionally, the increased $\mathrm{CH}_{4}$ mixing ratio leads to an increased $\mathrm{CO}_{2}$ production through photodissociation.

The response of several other chemical compounds (displayed in Fig. 20) to the doubling of the $\mathrm{CO}_{2}$ concentration can be explained as a combination of changes in rate constants and atmospheric density (due to the cooling), and in the strength of up- and downwelling. Under enhanced $\mathrm{CO}_{2}$ concentrations, the ratio between the $\mathrm{O}_{3}$ and $\mathrm{O}$ mixing ratios is generally shifted toward higher $\mathrm{O}_{3}$, which is a consequence of the strong temperature dependence of the ozone production reaction $\left(\mathrm{O}+\mathrm{O}_{2}+\mathrm{M} \rightarrow \mathrm{O}_{3}+\mathrm{M}\right)$. The large decrease in the atomic oxygen mixing ratio at high summer latitudes above $0.01 \mathrm{hPa}$ results from increased upwelling and leads also to an ozone decrease at this level. The ozone decrease in the polar winter around $0.1 \mathrm{hPa}(\sim 65$ $\mathrm{km}$ ) is mainly caused by the increase of $\mathrm{NO}$ and (to a lesser extent) $\mathrm{Cl}$ mixing ratios due to stronger subsidence of NO and Cl-rich air. The methane increase can be explained by the temperature dependence of the loss reactions with $\mathrm{OH}$ and chlorine. The increase in thermospheric water vapor is a consequence of the increased production by photodissociation of methane. It should be noted that the sometimes large relative changes in ozone, methane, and water vapor in the thermosphere are occurring where the absolute mixing ratios of these constituents are very low. The $\mathrm{OH}$ shows a strong response with changing sign at $\sim 0.01$ and $\sim 3.0$ $\times 10^{-5} \mathrm{hPa}(\sim 115 \mathrm{~km})$. At different heights, different production reactions are the main source of $\mathrm{OH}$, lead- 

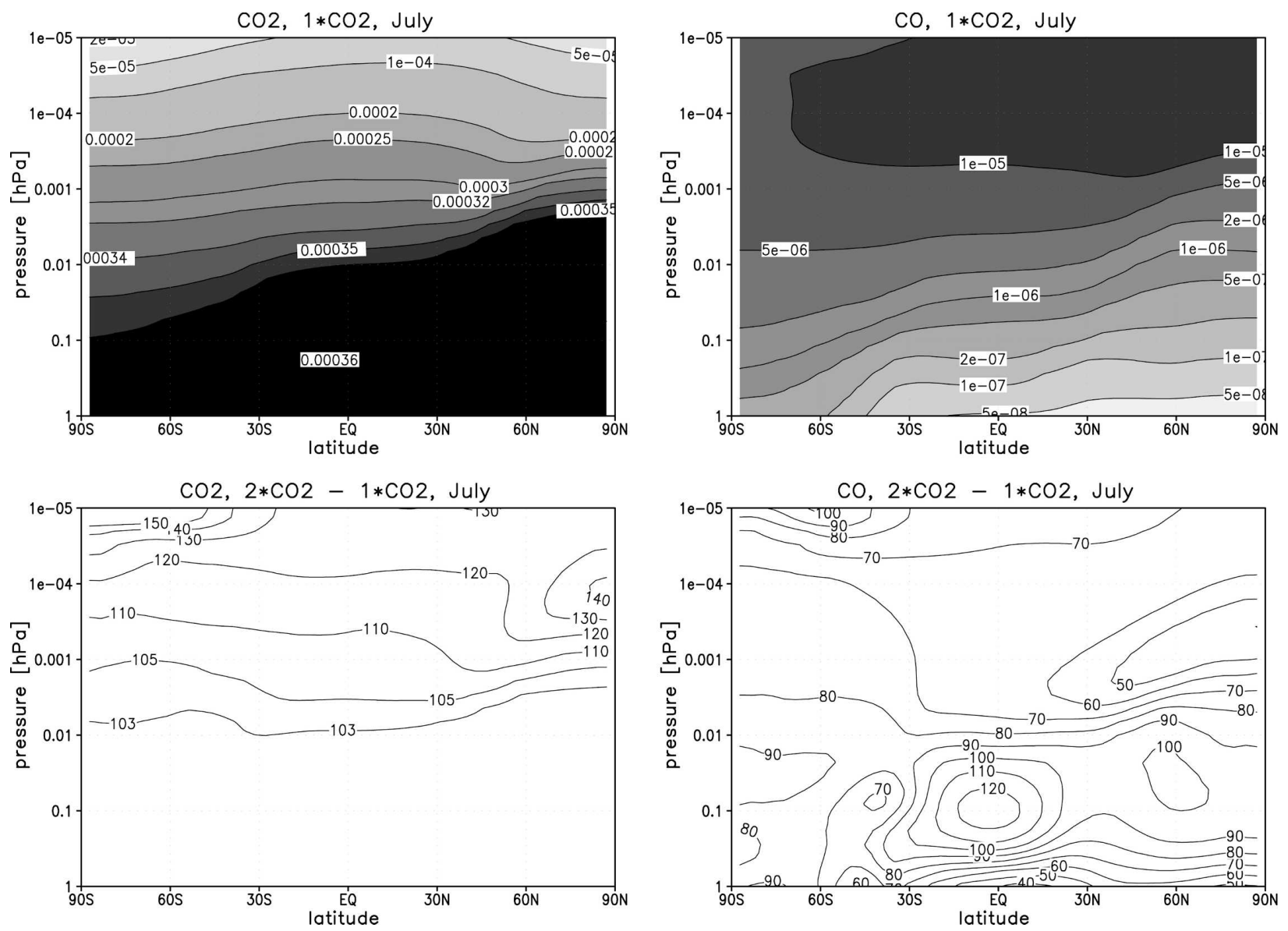

FIG. 19. Zonal mean July volume mixing ratios for $\mathrm{CO}_{2}$ and $\mathrm{CO}$ as simulated in the $\times \mathrm{CO}_{2}$ run, and the relative changes (\%) for the $2 \times \mathrm{CO}_{2}$ simulation with respect to $1 \times \mathrm{CO}_{2}$. The statistical significance of the changes is everywhere larger than $99 \%$.

ing to the change of signs: the $\mathrm{OH}$ increase above $\sim 0.01$ $\mathrm{hPa}$ is caused by the temperature dependence of the reaction $\mathrm{HO}_{2}+\mathrm{O} \rightarrow \mathrm{OH}+\mathrm{O}_{2}$ and by the ozone increase leading to more $\mathrm{OH}$ via the reaction $\mathrm{H}+\mathrm{O}_{3}$ $\rightarrow \mathrm{OH}+\mathrm{O}_{2}$. The reaction rate of $\mathrm{O}+\mathrm{H}_{2} \rightarrow \mathrm{OH}+\mathrm{H}$ is decreasing with decreasing temperature and therefore leading to the $\mathrm{OH}$ decrease above $\sim 3.0 \times 10^{-5}$ $\mathrm{hPa}$.

\section{b. Comparison with other models}

A variety of different models have been used in the past to study the effect of $\mathrm{CO}_{2}$ doubling. They have concentrated on temperature not only in the stratosphere but also in the mesosphere and thermosphere. Most of them are 2D (Portmann et al. 1995) or 3D (Rind et al. 1990; Berger and Dameris 1993; Akmaev and Fomichev 1998) dynamical models. The feedback on chemistry was included, for example, in a 1D model study by Roble and Dickinson (1989), in simulations with slightly different versions of the $2 \mathrm{D}$ model
SOCRATES by K2002 and by Gruzdev and Brasseur (2005), and in a 3D model study by Jonsson et al. (2004) covering heights up to approximately $100 \mathrm{~km}(\sim 2.0 \times$ $10^{-4} \mathrm{hPa}$ ). Most models derive a cooling of about $10 \mathrm{~K}$ (or more) near the stratopause and the mesopause, and a weaker cooling in the mesosphere. Our results suggest relatively low cooling rates around the mesopause probably due to the full inclusion in HAMMONIA of dynamical and chemical (increase of $\mathrm{O}_{3}$ ) feedbacks. This is in agreement with the study by Jonsson et al. (2004) that also includes the ozone feedback but no SST changes. The studies by Rind et al. (1990) and Portmann et al. (1995) point out the importance of these dynamical feedbacks. The increased residual circulation reported by Rind et al. (1990) is in general agreement with our simulations, as are the changes in the residual vertical velocity calculated by K2002. The chemical response to $\mathrm{CO}_{2}$ doubling presented by $\mathrm{K} 2002$ is relatively similar to our simulations for $\mathrm{O}_{3}, \mathrm{O}$, and $\mathrm{OH}$. In the case of $\mathrm{H}_{2} \mathrm{O}$ and $\mathrm{CH}_{4}$, both models show a 

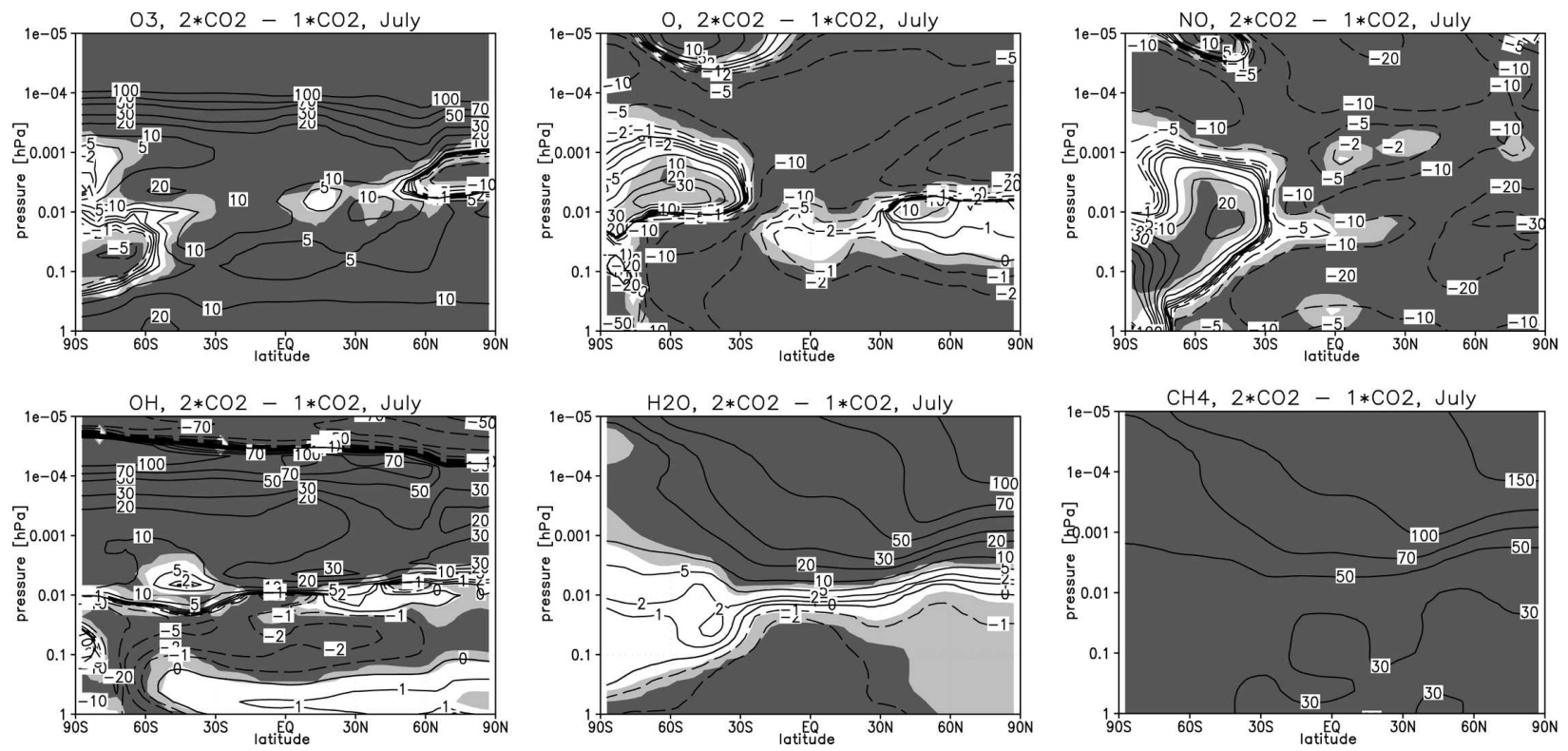

FIG. 20. Relative changes (\%) of July zonal mean volume mixing ratios for $\mathrm{O}_{3}, \mathrm{O}, \mathrm{NO}, \mathrm{OH}, \mathrm{H}_{2} \mathrm{O}$, and $\mathrm{CH}_{4}$ for the $2 \times \mathrm{CO}_{2}$ simulation with respect to $1 \times \mathrm{CO}_{2}$. Statistical significance of the changes that is larger than $90 \%$ (99\%) is indicated by light (dark) gray shading.

relatively strong increase of the respective mixing ratios in the lower thermosphere. However, in K2002, the water vapor response is positive in the entire mesosphere while, in the present study, it is weakly negative in the lower mesosphere. The $\mathrm{CH}_{4}$ response predicted by HAMMONIA is positive everywhere in the mesosphere, while in K2002 it is negative in the lower mesosphere. Jonsson et al. (2004) present similar percentage variations for the mesospheric ozone response in January as predicted by HAMMONIA for July, including relatively small areas of negative response at high latitudes in the upper mesosphere.

\section{Discussion and conclusions}

The results of this study have been presented using atmospheric pressure as the vertical coordinate. This is a natural choice because model levels (above $100 \mathrm{hPa}$ ) are levels of constant pressure. In addition, the interpretation of changes in the chemical composition is easier on constant pressure levels than in height coordinates. However, as most observations are performed with respect to geometric altitude, it is useful to present model results as well for constant height levels. It has been noted earlier in the context of $\mathrm{CO}_{2}$ doubling experiments (e.g., by Akmaev and Fomichev 1998) that atmospheric changes can be very different for fixed height and fixed pressure. This is a consequence of both a temperature (and subsequent density) change at one pressure level and the integrated effect of temperature changes below this level. Figure 1 shows that strong atmospheric expansion and contraction occurs for the experiments of this study. Figure 21 presents the effects of the solar cycle and of $\mathrm{CO}_{2}$ doubling on temperature and ozone mixing ratios in the mesosphere and lower thermosphere with respect to geometric height. For the solar cycle effect with only a moderate atmospheric expansion up to the mesopause, differences between pressure and height coordinates are relatively small. In the case of the temperature response, a weak vertical wave structure around the summer mesopause with a local minimum at $100 \mathrm{~km}\left(\sim 2.0 \times 10^{-4} \mathrm{hPa}\right)$ occurs only in height coordinates. The latitudinal gradient with a strong temperature response at high summer latitudes around $10^{-3} \mathrm{hPa}(\sim 92 \mathrm{~km}$; see Fig. 11) is not visible in height coordinates. In the case of ozone, there is a continuous pole-to-pole region of positive response near the mesopause that is interrupted when represented in pressure coordinates as described earlier. The effect of $\mathrm{CO}_{2}$ doubling on ozone appears as completely different in pressure coordinates (positive everywhere except at some altitudes in high-latitude regions) and in height coordinates (vertically alternating strong positive and negative responses). This latter pattern is mainly a consequence of large positive and negative vertical gradients in the ozone mixing ratios. The atmospheric cooling due to the $\mathrm{CO}_{2}$ increase exhibits a second minimum in a height region located near $110 \mathrm{~km}\left(\sim 5 \times 10^{-5} \mathrm{hPa}\right)$ while it is constantly increasing above the mesopause when displayed in pressure coordinates. However, the 

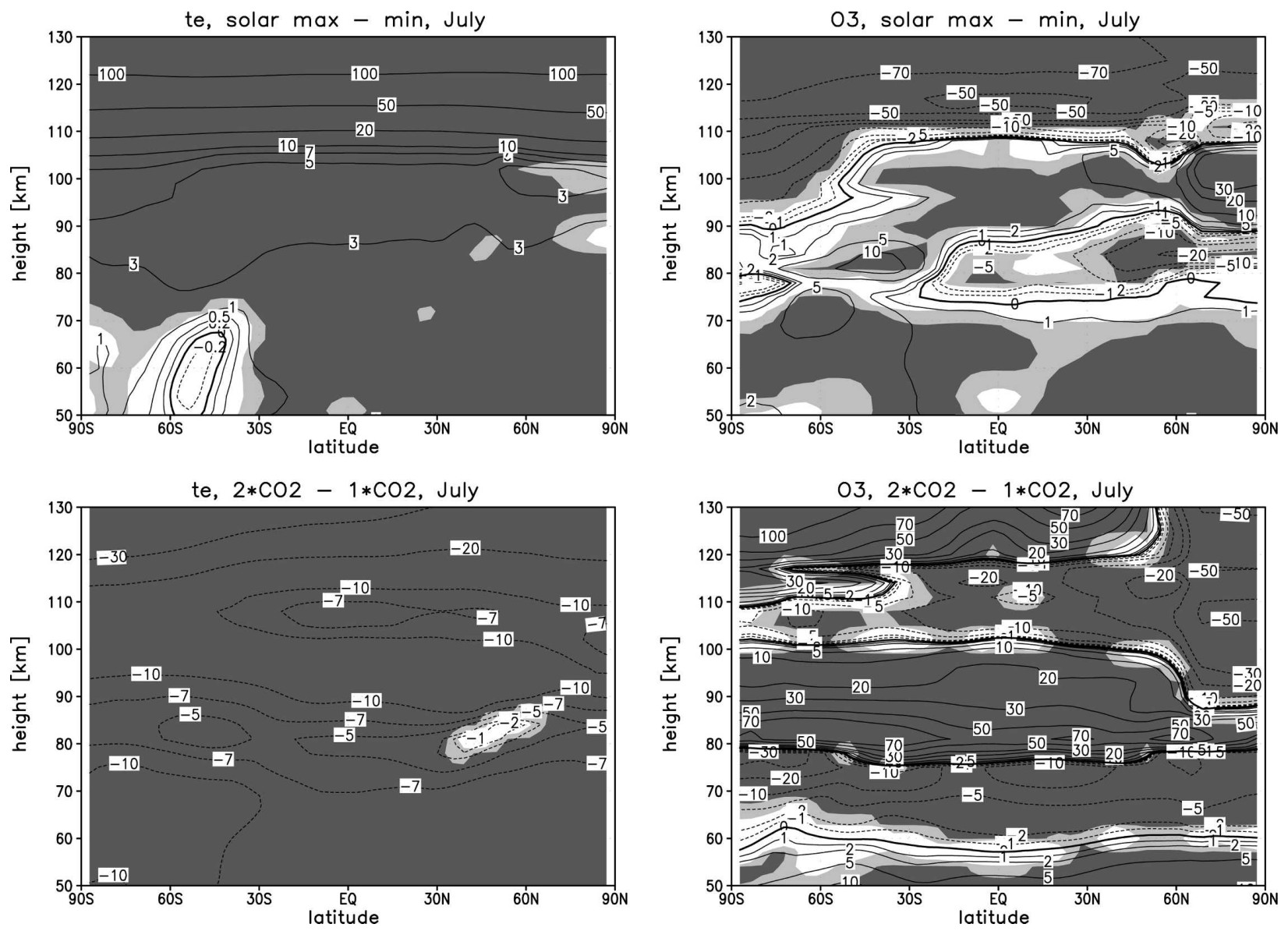

FIG. 21. Changes of July zonal mean temperature (K) and ozone (\%) (top) for the solar maximum simulation with respect to solar minimum and (bottom) for the $2 \times \mathrm{CO}_{2}$ simulation with respect to $1 \times \mathrm{CO}_{2}$. Please note that in contrast to all previous figures the changes are shown here with the geometrical height as vertical coordinate. For the comparisons of the same fields in pressure coordinates see Figs. 11, 12, 15, and 20.

temperature effect remains negative everywhere in the thermosphere, a result that differs from those obtained by Akmaev and Fomichev (1998) and Gruzdev and Brasseur (2005), who present a temperature increase in a part of the lower thermosphere. Reasons for these discrepancies to our results may be that the simulations by Akmaev and Fomichev (1998) do not include interactive chemistry so that a possible enhancement of the cooling by the more-than-doubled $\mathrm{CO}_{2}$ mixing ratio in the thermosphere could not be included. The relatively low upper lid at a log-pressure altitude of $120 \mathrm{~km}$ may represent an error source in the simulations of Gruzdev and Brasseur (2005). However, it should be noted that none of the models represents explicitly the NO cooling in the $5.3-\mu \mathrm{m}$ band, so that a potentially important feedback is missing.

Observational studies of mesospheric and thermospheric variations in relation with solar variability are rare. Beig et al. (2003), in their review paper on meso- spheric temperature trends, present a collection of different local analyses for the temperature response to the solar cycle. For the layer between $85-$ and $90-\mathrm{km}$ $\left(\sim 3 \times 10^{-3} \mathrm{hPa}\right)$ altitude, a relatively large number of observations exists. The responses lie between 0 and 5 $\mathrm{K}$ normalized to an irradiance change of 100 solar flux units (sfu; solar radiation at a wavelength of $10.7 \mathrm{~cm}$ in $10^{-22} \mathrm{~m}^{-2} \mathrm{~Hz}^{-1}$ ). The latitudinal differences in the response suggested by the observations cannot be confirmed by our simulations that give 2 to $2.5 \mathrm{~K}$ (100 $\mathrm{sfu})^{-1}$ at all latitudes. However, the errors associated with the analysis of the observations are in general large (see Beig et al. 2003, and references therein). DeLand et al. (2003) show a decrease in the occurrence frequency of polar mesospheric clouds (PMCs) for solar maximum conditions. This should theoretically either be related to an increase in temperature, a decrease in the water vapor concentration, or a decrease in the concentration of condensation nuclei in the polar 
summer mesopause region (or a combination of these causes). Our simulation suggests that at least the first two phenomena are occurring. Reliable analyses of the solar effect on other dynamical or chemical parameters are to our knowledge not available. It is difficult to evaluate the model results for a doubling of $\mathrm{CO}_{2}$ with observational data. However, if one assumes that the temperature trends observed for the past decades in the middle and upper atmosphere are mainly due to the $\mathrm{CO}_{2}$ increase, the lack of a detectable trend in the summer mesopause region (Beig et al. 2003) is consistent with our results for a $\mathrm{CO}_{2}$ doubling.

HAMMONIA represents a new generation of atmospheric models that try to combine classical general circulation models with comprehensive chemistry over a large altitude range. It aims at including all important atmospheric feedbacks in the model to make the simulations as realistic as possible. Running such a model has become possible with the increase in computer power made available during the last years. Nevertheless, it is not yet possible to run such a large model for very long time spans or make a large number of sensitivity tests. HAMMONIA will mainly be applied for research on vertical coupling phenomena in the atmosphere and for those cases where a simulation should be as realistic as possible, for example, for the explanation of observed mesospheric trends.

Despite the complexity of the model, we still see deficiencies that may have altered the results of this study. For example, the current model version does not simulate the QBO of equatorial stratospheric winds, which is believed to interact with the effect of solarinduced atmospheric variability (e.g., Labitzke 2003). This deficiency is expected to be overcome by increasing the vertical model resolution as was done in the MAECHAM5 model (Giorgetta et al. 2002). The present version of HAMMONIA is also lacking a representation of the role of ions. The abundance of ions depends on the intensity of solar irradiance and energetic particle fluxes and influences neutral chemistry in particular in the thermosphere and mesosphere.

This paper is intended to introduce a new model that extends from the surface to the thermosphere, evaluate its results by comparing them to available observations, and assess the atmospheric response to different types of external forcing that are classic benchmarks for such models. The model simulations have produced a huge amount of data. The basic analysis of the data has lead to the results presented in the present study. More detailed analyses of, for example, the energy budget or the response of tides and other waves to the different types of forcing will be the subject of subsequent papers.
Acknowledgments. The authors are grateful to Judith Lean, Naval Research Laboratory, Washington, DC, for providing the solar irradiance spectra. We also thank the partners of the Mesospheric Dynamics, Energetics and Chemistry (MEDEC) research project for help in the evaluation of HAMMONIA. We are grateful to the TIMED-SABER Science Team for providing access to data from the SABER instrument. The work was financed by the German Ministry of Education and Research (BMBF) within the framework of the AFO2000 research program under Contract 07ATF10. We also acknowledge the support of the German Climate Computing Center (DKRZ) where the numerical computations were performed. Finally, we thank two anonymous reviewers for their very helpful comments.

\section{REFERENCES}

Akmaev, R. A., and V. I. Fomichev, 1998: Cooling of the mesosphere and lower thermosphere due to doubling of $\mathrm{CO}_{2}$. Ann. Geophys., 16, 1501-1512.

— , V. A. Yudin, and D. A. Ortland, 1997: SMLTM simulation of the diurnal tide: Comparison with UARS observations. Ann. Geophys., 15, 1187-1197.

Allen, M., J. I. Lunne, and Y. L. Yung, 1984: The vertical distribution of ozone in the mesosphere and lower thermosphere. J. Geophys. Res., 89, 4841-4872.

Bailey, S. M., C. A. Barth, and S. C. Solomon, 2002: A model of nitric oxide in the lower thermosphere. J. Geophys. Res., 107, 1206, doi:10.1029/2001JA000258.

Banks, P. M., and G. Kockarts, 1973: Aeronomy, Part B. Academic Press, 355 pp.

Barth, C. A., K. D. Mankoff, S. M. Bailey, and S. C. Solomon, 2003: Global observations of nitric oxide in the thermosphere. J. Geophys. Res., 108, 1027, doi:10.1029/ 2002JA009458.

Batchelor, G. K., 1967: An Introduction to Fluid Dynamics. Cambridge University Press, 635 pp.

Beagley, S. R., J. de Grandpré, J. N. Koshyk, N. A. McFarlane, and T. G. Shepherd, 1997: Radiative-dynamical climatology of the first-generation Canadian middle atmosphere model. Atmos.-Ocean, 35, 293-331.

Beig, G., and Coauthors, 2003: Review of mesospheric temperature trends. Rev. Geophys., 41, 1015, doi:10.1029/ 2002RG000121.

Berger, U., and M. Dameris, 1993: Cooling of the mesosphere due to $\mathrm{CO}_{2}$ increases: A model study. Ann. Geophys., 11, 809819.

Boville, B. A., 1995: Middle atmosphere version of the CCM2 (MACCM2): Annual cycle and interannual variability. $J$. Geophys. Res., 100, 9017-9039.

Brasseur, G. P., and D. Offermann, 1986: Recombination of atomic oxygen near the mesopause: Interpretation of rocket data. J. Geophys. Res., 91, 10 818-10 824.

_ , and S. Solomon, 1986: Aeronomy of the Middle Atmosphere. D. Reidel, 452 pp.

- A. De Rudder, G. M. Keating, and J. Nicholson III, 1987: Response of middle atmosphere to short-term solar ultraviolet variations: 2. Theory. J. Geophys. Res., 92, 903-914.

_ _ X. Tie, P. J. Rasch, and F. Lefvre, 1997: A three-dimensional 
model simulation of the antarctic ozone hole: Impact of anthropogenic chlorine on the lower stratosphere and upper troposphere. J. Geophys. Res., 102, 8909-8930.

Chabrillat, S., and G. Kockarts, 1998: Correction to "Simple parameterization of the absorption of the solar lyman-alpha line." Geophys. Res. Lett., 25, 79-80.

- — - D. Fonteyn, and G. Brasseur, 2002: Impact of molecular diffusion on the $\mathrm{CO}_{2}$ distribution and the temperature in the mesosphere. Geophys. Res. Lett., 29, 1729, doi:10.1029/ 2002 GL015309.

Charron, M., and E. Manzini, 2002: Gravity waves from fronts: Parameterization and middle atmosphere response in a general circulation model. J. Atmos. Sci., 59, 923-941.

Chen, L., J. London, and G. Brasseur, 1997: Middle atmospheric ozone and temperature responses to solar irradiance variations over 27-day periods. J. Geophys. Res., 102, $29957-$ 29979.

Chipperfield, M. P., D. Cariolle, P. Simon, R. Ramaroson, and D. J. Lary, 1993: A 3-dimensional modeling study of trace species in the arctic lower stratosphere during winter 19891990. J. Geophys. Res., 98, 7199-7218.

de Grandpré, J., S. R. Beagly, V. I. Fomichev, E. Griffioen, J. C. McConnell, and A. S. Medvedev, 2000: Ozone climatology using interactive chemistry: Results from the Canadian middle atmosphere model. J. Geophys. Res., 105, $26475-$ 26491.

DeLand, M. T., E. P. Shettle, G. E. Thomas, and J. J. Olivero, 2003: Solar backscattered ultraviolet (SBUV) observations of polar mesospheric clouds (PMCs) over two solar cycles. $J$. Geophys. Res., 108, 8445, doi:10.1029/2002JD002398.

Fels, S. B., J. D. Mahlman, M. D. Schwarzkopf, and R. W. Sinclair, 1980: Stratospheric sensitivity to perturbations in ozone and carbon dioxide: Radiative and dynamical response. $J$. Atmos. Sci., 37, 2265-2297.

Fomichev, V. I., and J.-P. Blanchet, 1995: Development of the new CCC/GCM longwave radiation model for extension into the middle atmosphere. Atmos.-Ocean, 33, 513-531.

, — , and D. S. Turner, 1998: Matrix parameterization of the $15 \mu \mathrm{m} \mathrm{CO}$ band cooling in the middle and upper atmosphere for variable $\mathrm{CO}_{2}$ concentration. J. Geophys. Res., 103, 11 505-11 528.

, W. E. Ward, S. R. Beagley, C. McLandress, J. C. McConnell, N. A. McFarlane, and T. G. Shepherd, 2002: Extended Canadian Middle Atmosphere Model: Zonal mean climatology and physical parameterizations. J. Geophys. Res., 107, 4087, doi:10.1029/2001JD000479.

— C. Fu, J. de Grandpré, S. R. Beagley, V. P. Ogibalov, and J. C. McConnell, 2004a: Model thermal response to minor radiative energy sources and sinks in the middle atmosphere. J. Geophys. Res., 109, D19107, doi:10.1029/2004JD004892.

— , V. P. Ogibalov, and S. R. Beagley, 2004b: Solar heating by the near-IR $\mathrm{CO}_{2}$ bands in the mesosphere. Geophys. Res. Lett., 31, L21102, doi:10.1029/2004GL020324.

Fouquart, Y., and B. Bonnel, 1980: Computations of solar heating of the earth's atmosphere: A new parameterization. Contrib. Atmos. Phys., 53, 35-62.

Garcia, R. R., 2000: The role of equatorial waves in the semiannual oscillation of the middle atmosphere. Atmospheric Science across the Stratopause, Geophys. Monogr., Vol. 123, Amer. Geophys. Union, 161-176.

, T. J. Dunkerton, R. S. Lieberman, and R. A. Vincent, 1997: Climatology of the semiannual oscillation of the tropical middle atmosphere. J. Geophys. Res., 102, 26 019-26 032.
Gettelman, A., D. E. Kinnison, T. J. Dunkerton, and G. P. Brasseur, 2004: The impact of monsoon circulations on the upper troposphere and lower stratosphere. J. Geophys. Res., 109, D22101, doi:10.1029/2004JD004878.

Gill, A. E., 1982: Atmosphere-Ocean Dynamics. Academic Press, $662 \mathrm{pp}$.

Giorgetta, M. A., E. Manzini, and E. Roeckner, 2002: Forcing of the quasi-biennial oscillation from a broad spectrum of atmospheric waves. Geophys. Res. Lett., 29, 1245, doi:10.1029/ 2002GL014756.

,,--- M. Esch, and L. Bengtsson, 2006: Climatology and forcing of the quasi-biennial oscillation in the MAECHAM5 model. J. Climate, 19, 3882-3901.

Gruzdev, A. N., and G. P. Brasseur, 2005: Long-term changes in the mesosphere calculated by a two-dimensional model. $J$. Geophys. Res., 110, D03304, doi:10.1029/2003JD004410.

Hamilton, K., R. J. Wilson, J. D. Mahlman, and L. J. Umscheid, 1995: Climatology of the SKYHI troposphere-stratospheremesosphere general circulation model. J. Atmos. Sci., 52, 5-43.

Heaps, M. G., 1978: A parameterization of cosmic ray ionization. Planet. Space Sci., 26, 513-517.

Hedin, A., 1991: Extension of the MSIS thermosphere model into the middle and lower atmosphere. J. Geophys. Res., 96, 11591172.

Hines, C. O., 1997a: Doppler-spread parameterization of gravity wave momentum deposition in the middle atmosphere. Part 1: Basic formulation. J. Atmos. Sol. Terr. Phys., 59, 371-386.

_, 1997b: Doppler-spread parameterization of gravity wave momentum deposition in the middle atmosphere. Part 2: Broad and quasi monochromatic spectra, and implementation. J. Atmos. Sol. Terr. Phys., 59, 387-400.

Holton, J. R., and M. J. Alexander, 2000: The role of waves in the transport circulation of the middle atmosphere. Atmospheric Science across the Stratopause, Geophys. Monogr., Vol. 123, Amer. Geophys. Union, 21-36.

Hong, S. S., and R. S. Lindzen, 1976: Solar semidiurnal tide in the thermosphere. J. Atmos. Sci., 33, 135-153.

Horowitz, L. W., and Coauthors, 2003: A global simulation of tropospheric ozone and related tracers: Description and evaluation of MOZART, version 2. J. Geophys. Res., 108, 4784, doi:10.1029/2002JD002853.

Huang, T. Y. W., and G. P. Brasseur, 1993: Effect of long-term solar variability in a two-dimensional interactive model of the middle atmosphere. J. Geophys. Res., 98, 20 412-20 427.

—_, and Coauthors, 1998: Description of SOCRATES-A chemical dynamical radiative two-dimensional model. Tech. Rep. NCAR/TN-440+EDD, NCAR, Boulder, CO, 94 pp.

Jonsson, A. I., J. de Grandpré, V. I. Fomichev, J. C. McConnell, and S. R. Beagley, 2004: Doubled $\mathrm{CO}_{2}$-induced cooling in the middle atmosphere: Photochemical analysis of the ozone radiative feedback. J. Geophys. Res., 109, D24103, doi:10.1029/ 2004JD005093.

Kaufmann, M., O. A. Gusev, K. U. Grossmann, F. J. MartinTorres, D. R. Marsh, and A. A. Kutepov, 2003: Satellite observations of daytime and nighttime ozone in the mesosphere and lower thermosphere. J. Geophys. Res., 108, 4272, doi:10.1029/2002JD002800.

Khosravi, R., G. Brasseur, A. Smith, D. Rusch, S. Walters, S. Chabrillat, and G. Kockarts, 2002: Response of the mesosphere to human-induced perturbations and solar variability calculated by a 2-d model. J. Geophys. Res., 107, 4358, doi:10.1029/2001JD001235. 
Koppers, G. A. A., and D. P. Murtagh, 1996: Model studies of the influence of $\mathrm{O}_{2}$ photodissociation parameterizations in the Schumann-Runge bands on ozone related photolysis in the upper atmosphere. Ann. Geophys., 14, 68-79.

Labitzke, K., 2003: The global signal of the 11-year sunspot cycle in the atmosphere: When do we need the QBO? Meteor. Z., 12, 209-216.

Lean, J., J. Rottman, G. J. Kyle, H. L. Woods, T. N. Hickey, and J. R. Pugga, 1997: Detection and parameterization of variations in solar mid and near-ultraviolet radiation (200-400 nm). J. Geophys. Res., 102, 29 939-29956.

Lefèvre, F., G. Brasseur, I. Folkins, and A. K. Smith, 1994: Stratospheric chlorine monoxide and ozone: Three-dimensional model simulations. J. Geophys. Res., 99, 8183-8195.

Lieberman, R. S., and P. B. Hays, 1994: An estimate of the momentum deposition in the lower thermosphere by the observed diurnal tide. J. Atmos. Sci., 51, 3094-3105.

Lin, S.-J., and R. B. Rood, 1996: Multidimensional flux-form semi-Lagrangian transport schemes. Mon. Wea. Rev., 124, 2046-2070.

Lott, F., and M. J. Miller, 1997: A new subgrid-scale orographic drag parameterization: Its formulation and testing. Quart. J. Roy. Meteor. Soc., 123, 101-127.

Madronich, S., and S. Flocke, 1998: The role of solar radiation in atmospheric chemistry. Handbook of Environmental Chemistry, P. Boule, Ed., Springer, 1-26.

Manzini, E., N. A. McFarlane, and C. McLandress, 1997: Impact of the Doppler spread parameterization on the simulation of the middle atmosphere circulation using the MA/ECHAM4 general circulation model. J. Geophys. Res., 102, $25751-$ 25762.

- M. A. Giorgetta, M. Esch, L. Kornblueh, and E. Roeckner, 2006: The influence of sea surface temperatures on the Northern winter stratosphere: Ensemble simulations with the MAECHAM5 model. J. Climate, 19, 3863-3881.

Marsh, D., A. Smith, G. Brasseur, M. Kaufmann, and K. Grossmann, 2001: The existence of a tertiary ozone maximum in the high-latitude middle mesosphere. Geophys. Res. Lett., 28, 4531-4534.

,-- , and E. Noble, 2003: Mesospheric ozone response to changes in water vapor. J. Geophys. Res., 108, 4109, doi:10.1029/2002JD002705.

— S. C. Solomon, and A. E. Reynolds, 2004: Empirical model of nitric oxide in the lower thermosphere. J. Geophys. Res., 109, A07301, doi:10.1029/2003JA010199.

Marsland, S. J., H. Haak, J. H. Jungclaus, M. Latif, and F. Röske, 2003: The Max-Planck-Institute global ocean/sea ice model with orthogonal curvilinear coordinates. Ocean Modell., 5, 91-127.

McEwan, M. J., and L. F. Phillips, 1975: Chemistry of the Atmosphere. Edward Arnold Ltd., 301 pp.

Mertens, C. J., M. G. Mlynczak, M. Lopez-Puertas, P. P. Wintersteiner, R. H. Picard, J. R. Winick, L. L. Gordley, and J. M. Russell III, 2001: Retrieval of mesospheric and lower thermospheric kinetic temperature from measurements of $\mathrm{CO}_{2}$ $15 \mu \mathrm{m}$ earth limb emission under non-LTE conditions. Geophys. Res. Lett., 28, 1391-1394.

— temperatures and comparisons with falling sphere measurements taken during the 2002 summer MaCWAVE campaign. Geophys. Res. Lett., 31, L03105, doi:10.1029/2003GL018605.

Minschwaner, K., and D. E. Siskind, 1993: A new calculation of
NO photolysis in the stratosphere, mesosphere, and lower thermosphere. J. Geophys. Res., 98, 20 401-20 412.

Miyahara, S., Y. Yoshida, and Y. Miyoshi, 1993: Dynamic coupling between the lower and upper atmosphere by tides and gravity waves. J. Atmos. Terr. Phys., 55, 1039-1053.

Mlawer, E. J., S. J. Taubman, P. D. Brown, M. J. Iacono, and S. J. Clough, 1997: Radiative transfer for inhomogeneous atmospheres: RRTM, a validated correlated-k model for the longwave. J. Geophys. Res., 102, 16 663-16 682.

Mlynczak, M. G., 2000: A contemporary assessment of the mesospheric energy budget. Atmospheric Science across the Stratopause, Geophys. Monogr., Vol. 123, Amer. Geophys. Union, $37-52$.

_ , and S. Solomon, 1993: A detailed evaluation of the heating efficiency in the middle atmosphere. J. Geophys. Res., 98, $10517-10541$.

Mote, P. W., and Coauthors, 1996: An atmospheric tape recorder: The imprint of tropical tropopause temperatures on stratospheric water vapor. J. Geophys. Res., 101, 3989-4006.

Ogibalov, V. P., and V. I. Fomichev, 2003: Parameterization of solar heating by the near IR $\mathrm{CO}_{2}$ bands in the mesosphere. Adv. Space Res., 32, 759-764.

Portmann, R. W., G. E. Thomas, S. Solomon, and R. R. Garcia, 1995: The importance of dynamical feedbacks on doubled $\mathrm{CO}_{2}$-induced changes in the thermal structure of the mesosphere. Geophys. Res. Lett., 22, 1733-1736.

Randel, W., F. Wu, J. M. Russell III, A. Roche, and J. W. Waters, 1998: Seasonal cycles and QBO variations in stratospheric $\mathrm{CH}_{4}$ and $\mathrm{H}_{2} \mathrm{O}$ observed in UARS HALOE data. J. Atmos. Sci., 55, 163-185.

Rasch, P. J., B. A. Boville, and G. R. Brasseur, 1995: A threedimensional general circulation model with coupled chemistry for the middle atmosphere. J. Geophys. Res., 100, 90419071.

Richards, P. G., J. A. Fennelly, and D. G. Torr, 1994: A solar EUV flux model for aeronomic calculations. J. Geophys. Res., 99, 8981-8992; Correction, 99, 13283.

Rind, D., R. Suozzo, N. K. Balachandran, and M. J. Prather, 1990: Climate change and the middle atmosphere. Part I: The doubled $\mathrm{CO}_{2}$ climate. J. Atmos. Sci., 47, 475-494.

Roble, R. G., 1995: Energetics of the mesosphere and thermosphere. The Upper Mesosphere and Lower Thermosphere: A Review of Experiment and Theory, Geophys. Monogr., Vol. 87, Amer. Geophys. Union, 1-21.

_ 2000: On the feasability of developing a global atmospheric model extending from the ground to the exosphere. Atmospheric Science across the Stratopause, Geophys. Monogr., Vol. 123, Amer. Geophys. Union, 53-68.

— , and R. E. Dickinson, 1989: How will changes in carbon dioxide and methane modify the mean structure of the mesosphere and thermosphere? Geophys. Res. Lett., 16, 14411444.

Roeckner, E., and Coauthors, 2003: The atmospheric general circulation model ECHAM 5. Part I: Model description. Tech. Rep. 349, MPI for Meteorology, Hamburg, Germany, 127 pp. , and Coauthors, 2005: The atmospheric general circulation model ECHAM 5. Part II: Sensitivity of simulated climate to horizontal and vertical resolution. Tech. Rep. 354, MPI for Meteorology, Hamburg, Germany, 64 pp.

— , and Coauthors, 2006: Sensitivity of simulated climate to horizontal and vertical resolution in the ECHAM5 atmosphere model. J. Climate, 19, 3771-3791.

Rose, K., and G. Brasseur, 1989: A three-dimensional model of 
chemically active trace species in the middle atmosphere during disturbed winter conditions. J. Geophys. Res., 94, $16387-$ 16403.

Rozanov, E. V., M. E. Schlesinger, T. A. Egorova, B. Li, N. Andronova, and V. A. Zubov, 2004: Atmospheric response to the observed increase of solar UV radiation from solar minimum to solar maximum simulated by the University of Illinois at Urbana-Champaign climate-chemistry model. J. Geophys. Res., 109, D01110, doi:10.1029/2003JD003796.

Sassi, F., R. R. Garcia, B. A. Boville, and H. Liu, 2002: On temperature inversions and the mesospheric surf zone. J. Geophys. Res., 107, 4380, doi:10.1029/2001JD001525.

Simmons, A. J., D. M. Burridge, M. Jarraud, C. Girard, and W. Wergen, 1989: The ECMWF medium-range prediction model: Development of the numerical formulations and the impact of increased resolution. Meteor. Atmos. Phys., 40, 28-60.

Smith, A. K., 2004: Physics and chemistry of the mesopause region. J. Atmos. Sol. Terr. Phys., 66, 839-857.

Sonnemann, G., C. Kremp, A. Ebel, and U. Berger, 1998: A threedimensional dynamic model of the minor constituents of the mesosphere. Atmos. Environ., 32, 3157-3172.

Steil, B., C. Brühl, E. Manzini, P. J. Crutzen, J. Lelieveld, P. J. Rasch, E. Roeckner, and K. Krüger, 2003: A new interactive chemistry-climate model: 1 . Present-day climatology and interannual variability of the middle atmosphere using the model and 9 years of HALOE/UARS data. J. Geophys. Res., 108, 4290, doi:10.1029/2002JD002971.

Summers, M. E., D. F. Strobel, R. M. Bevilacqua, X. Zhu, M. T. DeLand, M. Allen, and G. M. Keating, 1990: A model study of the response of mesospheric ozone to short-term solar ultraviolet flux variations. J. Geophys. Res., 95, 22523 22538.

Swinbank, R., and D. A. Ortland, 2003: Compilation of wind data for the Upper Atmosphere Research Satellite (UARS) Reference Atmosphere Project. J. Geophys. Res., 108, 4615, doi:10.1029/2002JD003135.

von Zahn, U., and U. Berger, 2003: Persistent ice cloud in the midsummer upper mesosphere at high latitudes: Threedimensional modeling and cloud interactions with ambient water vapor. J. Geophys. Res., 108, 8451, doi:10.1029/ 2002JD002409.

Wang, H. J., D. M. Cunnold, and X. Bao, 1996: A critical analysis of stratospheric aerosol and gas experiment ozone trends. $J$. Geophys. Res., 101, 12 495-12 514.

Zhu, X., J.-H. Yee, and E. R. Talaat, 2003: Effect of short-term solar ultraviolet flux variability in a coupled model of photochemistry and dynamics. J. Atmos. Sci., 60, 491-509. 